Nonlinear Processes in Geophysics (2001) 8: 223-239

Nonlinear Processes

in Geophysics

(C)European Geophysical Society 2001

\title{
Generation of second mode solitary waves by the interaction of a first mode soliton with a sill
}

\author{
V. I. Vlasenko and K. Hutter \\ Institut für Mechanik, Technische Universität Darmstadt, Hochschulstr. 1, D-64289 Darmstadt, Germany
}

Received: 10 July 2000 - Accepted: 18 September 2000

\begin{abstract}
Results of an experimental and theoretical study of the interaction of a first mode internal solitary wave with a localised bottom topography (sill) are presented. Laboratory experiments have been performed in a $10 \mathrm{~m}$ long and $0.33 \mathrm{~m}$ wide channel filled with a stratified fluid. The interface between the two layers (fresh and salt water) is diffuse and has a finite thickness. Soliton-type disturbances of the interface having characteristics of the first baroclinic mode are generated at one channel end. They move along the channel and encounter an underwater obstacle (sill) in the middle of the channel, where they break into reflected and transmitted waves. Two types of internal waves are produced by the interaction: a fast first mode internal soliton and a slower (by a factor of approximately 3 ) second mode soliton-like wave.

A numerical model, based on the two-dimensional NavierStokes equations in the Boussinesq approximation, is used to reproduce the laboratory experiment. The detailed analysis of the horizontal and vertical structures of transmitted and reflected waves showed that the fast reflected and transmitted waves observed in the experiment can be interpreted as a first mode internal solitary wave whose characteristics are very close to those of the $\mathrm{K}-\mathrm{dV}$ solitons. It is also demonstrated that the slow speed waves, generated during the interaction behind the first fast wave have vertical and horizontal structures very close to the second mode internal K-dV solitons.
\end{abstract}

\section{Introduction}

Numerous in-situ and remote-sensing observations demonstrate the evidence of packets of long first-mode internal waves in marginal seas, straits, fjords and coastal waters (Osborne and Burch, 1980; Apel et al., 1985, 1995; Sandstrom and Elliot, 1984; Haury et al., 1979; Holloway, 1987; Liu, 1988). Such waves may evolve from disturbances caused

Correspondence to: K. Hutter

(hutter@mechanik.tu-darmstadt.de) by tidal flow over topography (continental slopes, oceanic banks, sills, and ridges). For a detailed review of these investigations one can refer to Ostrovsky and Stepanynts (1989). In lakes, first-mode internal wave packets can be excited in response to strong wind events (Farmer, 1978; Stevens et al., 1996). Probably such waves are an important part of energy transfer from large scales down to small ones (Wunsch and Munk, 1998); they contribute to the vertical mixing, while propagating at the interface where a shear layer develops. The shoreward propagating waves eventually break in a coastal zone where they are thought to be responsible for nutrient mixing (Sandstrom and Elliott, 1984).

Solitary waves may emerge if the propagation distance of a disturbance is sufficiently large. Once generated by a tidal flux over the shelf-edges, ridges or sills, they generally propagate for a large distance before encountering any further significant variation in bottom topography. Of course, the effects of dissipation and topography are important in determining the evolution of the wave. While the mechanism of generation of solitary internal waves is well recognised (Maxworthy, 1979; Lamb K.G., 1994; Gerkema and Zimmerman, 1994), their ultimate fate when solitary waves propagate towards the shore into shoaling regions remains comparatively poorly understood. At the same time there are a lot of experimental examples where the shoaling effects and local change of the bottom topography can influence the evolution and dynamics of solitary internal waves. We mention the observations of internal waves in the Andaman Sea (Osborne and Burch, 1980) and the Sulu Sea (Apel et al., 1985), in the Massachusetts Bay (Halpern, 1971) and on the Australian North West shelf (Holloway et al., 1997). Similar phenomena, when internal wave groups move towards the coastline, have been measured in lakes (Thorpe, 1971).

The dissipation of internal solitary waves propagating in the ocean of variable depth may occur through boundary layer viscosity, scattering by bottom topography and wave breaking. Most nonlinear internal waves observed in seas, straits and lakes were the first baroclinic mode depression waves. Nevertheless such waves reverse their polarity on 
passing through a "turning point" where the pycnocline is located approximately midway between the surface and the bottom and where the coefficient of nonlinearity changes its sign (Kaup and Newell, 1978). Such experimental data were obtained in the Rio-Ontario Strait (western Greece). In this place, two moving internal wave packets, consisting of elevation and depression waves separated by a 12-hour tidal period, were observed (Salusti et al., 1989). The idea of changing the polarity of solitary waves after they pass through a "turning point" was used in a paper by Liu et al. (1998) for the interpretation of SAR images south of Taiwan in the East China Sea and east of Huinan in the South China Sea. So this is a common situation in coastal regions, where a solitary wave of depression may encounter a "turning point" somewhere on the slope; first it will disintegrate into a dispersive wave train and then evolve to a packet of elevation waves in the shallow water area.

Several studies (theoretical, experimental) have been carried out on the propagation of solitary waves over a bottom topography. In Helfrich et al. (1984) the scattering of solitary waves in a two-layer system by a gradually varying change in the depth was theoretically investigated. It was found that more than one wave of reversed polarity may emerge as the incident wave passes through a "turning point". In a further paper (Helfrich and Melville, 1986) the evolution of a long solitary internal wave over the bottom topography was examined by a combination of laboratory experiment and theoretical modelling. Weak shearing and strong breaking (overturning) instabilities depending on the incident wave amplitude and stratification were observed in the experiments. In some cases the instability of the first baroclinic mode wave led to the generation of a second mode solitary wave. The breaking of an internal solitary wave of large amplitude over the sloping bottom was recently studied by laboratory experiments (Helfrich, 1992). It was found that shoaling of a solitary internal wave can result in wave breaking and the production of multiple soliton-like waves of elevation (turbulent surges or boluses) which propagate up the slope. This work on the dynamics (breaking and mixing) of solitary internal waves over an inclined bottom topography, which possessed relatively small amplitudes and small slopes $\left(<4^{\circ}\right)$, was continued for a wider range of amplitudes and slopes in a paper by Michallet and Ivey (1999).

Laboratory experiments on the interaction of solitary internal waves with a sill were also performed to study the energy loss caused by the interaction (Diebels et al., 1994; Maurer et al., 1996; Wessels and Hutter, 1996). It was found that the ratio of the height of the sill to the thickness of the lower layer was the parameter controlling the amount of transmitted and reflected energy. The theoretical background in that study was based on the weakly nonlinear theory for a two-layer fluid which resolves only the first baroclinic mode. Nevertheless, in a more recent paper (Hüttemann and Hutter, 2001) it was demonstrated that, with the help of laboratory experiments in a two-layer system (fresh water, salt water) in which the interface is not sharp but slightly diffuse, not only first but also the second mode solitary waves (reflected, transmitted) may be generated due to the interaction of the first mode internal soliton with a sill. This result was obtained on the basis of the analysis of the phase speed of the reflected waves from the sill and those transmitted. The possibility of the generation of second mode solitary waves, due to the interaction of the first mode solitons with the bottom topography, was also previously indicated in experimental work (Helfrich and Melville, 1986; Helfrich, 1992); furthermore, there are also some in-situ measurements of such waves (Mortimer, 1952; Salvade et al.,1987; Sabinin, 1992).

So, the goal of the present paper is (i) to build the mathematical model of the interaction of solitary internal waves with a sill in the frame of the Navier-Stokes equations for a continuously stratified fluid, and (ii) to study in detail the dynamics of the interaction of solitary waves with a sill in order to answer the question of the possibility of generation of second mode solitons during such interactions: this was formulated as a hypothesis in the paper of Hüttemann and Hutter (2001) on the basis of laboratory experiments. The paper is organised as follows. A short description of experimental data, obtained by Hüttemann and Hutter (2001) is presented in Sect. 2. Here we describe the experimental setup of the wave channel employed and measuring technique (Sect. 2.1). Then, Sect. 2.2 presents the experimental finding of possible generation of second mode solitary waves during interaction of the first mode solitons with a sill. Section 3 is devoted to a description of the theoretical formulation of the wave process in the channel, based on the Navier-Stokes equations. Governing equations, boundary conditions and numerical scheme are presented in Sect. 3.1 and then, in Sect. 3.2, the initialization of the model (background fluid stratification and initial conditions) are discussed . In particular, the analytic solution of the Korteveg-de Vries equation (hereafter K-dV) for stationary solitary waves in case of a smooth pycnocline is presented and its use in finding the initial wave field is discussed. The results of the numerical experiments on the interaction of the internal solitary wave with a sill are presented in Sect. 4 in four parts. First, in Sect. 4.1 the basic case run with the governing parameters reproducing the laboratory experiment of Sect. 2 is described in detail. Next, the influence of the blocking parameter $B$ (the dimensionless height of the sill), the Froude number $\mathrm{Fr}$ (the amplitude of the incident wave) and the viscosity are discussed in Sects. 4.2-4. A summary and conclusions are given in Sect. 5.

\section{The experimental data}

\subsection{Experimental setup and measuring technique}

The experimental arrangement and measuring techniques have been reported earlier in Diebels et al. (1994), Maurer et al. (1996), and Wessels and Hutter (1996). For details of the experimental setup and methods of measurements (the construction of the wave channel, wave generator, error estimation, performance of the experiments) the reader is referred to these papers. Here we shall describe briefly the experimental 


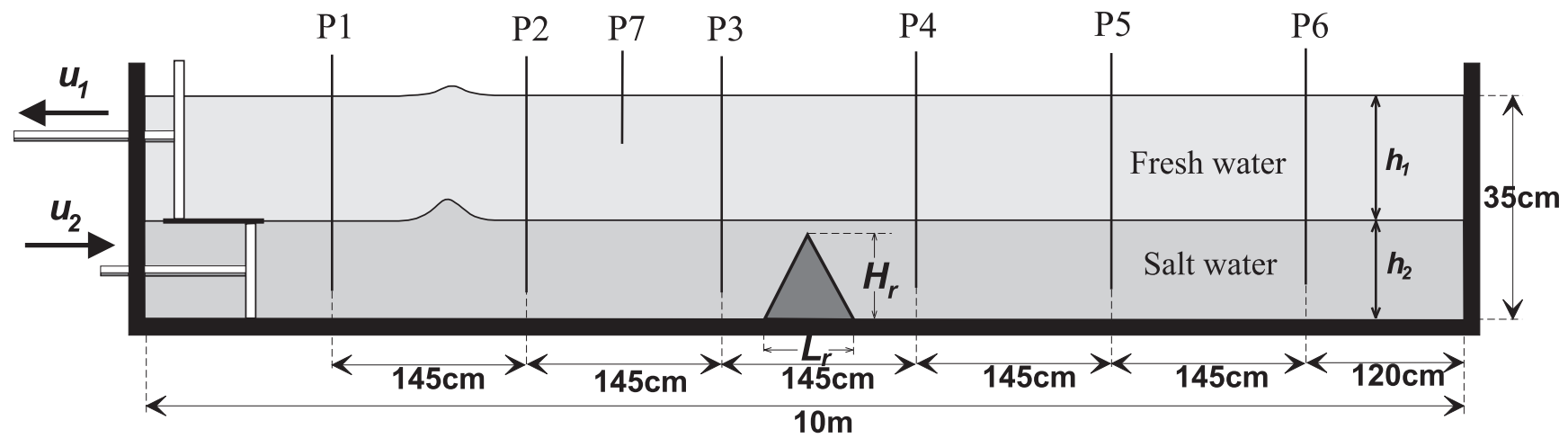

Fig. 1. Sketch of the experimental arrangement (frontal view). On the left are two pistons, separated by a thin plate at the interface level, which simultaneously move in opposite directions with velocities $u_{1}, u_{2}$ and displace the same volume of water. Six electrical resistivity gauges P1-P6 record the interface elevation. A seventh gauge P7 records the free surface motion. The triangular sill with the height $H_{r}$ and width $L_{r}$ is placed in the middle of the tank.

setup and typical experimental data obtained by Hüttemann and Hutter (2001). Experiments were conducted in a glasswalled wave tank $10 \mathrm{~m}$ long, $33 \mathrm{~cm}$ wide and $35 \mathrm{~cm}$ high. The schematic sketch of the experimental arrangement with the explanations of all basic parameters is presented in Fig. 1.

A salt-stratified system was constructed by filling the tank with fresh water (of density $10^{3} \mathrm{~kg} \mathrm{~m}^{-3}$ ) in the upper layer and then cautiously pouring salt water below (approximately $1022.5 \mathrm{~kg} \mathrm{~m}^{-3}$ ). The result is a two-layered configuration with a steep density transition through a diffuse interface (Fig. 2).

Internal water waves, excited by a wave generator at the left, travel down the channel to the right. The wave generator consists of two pistons at the heights of the two water layers and covering the whole width of the channel; they are moved in opposite directions in such a way that the transported water volume is the same in both layers $\left(u_{1} h_{1}=-u_{2} h_{2}\right)$. The main idea in generating solitary waves is to produce a positive definite elevation of a soliton-like shape that will then develop to a solitary wave during propagation. To achieve pure baroclinic waves, free surface elevations should be kept as small as possible.

The internal wave propagation and its interaction with built-in sills are measured by six gauges (P1-P6) along the channel. An additional surface gauge (P7) is installed to ensure that there is no significant surface elevation. The gauges consist of two parallel wires subjected to an alternating voltage and held in a vertical position in the middle of the channel width. The gauge signal is amplified and digitised for computational analysis. The gauges are attached to a vertical movable fixture, which is adjustable with a $0.1 \mathrm{~mm}$ resolution.

\subsection{The experiments}

The experiments investigated the interaction of solitary waves with a sill, built on the ground of the channel (Fig. 1). When partially blocking the lower layer by a sill an incoming solitary wave will be split into a reflected and a transmitted

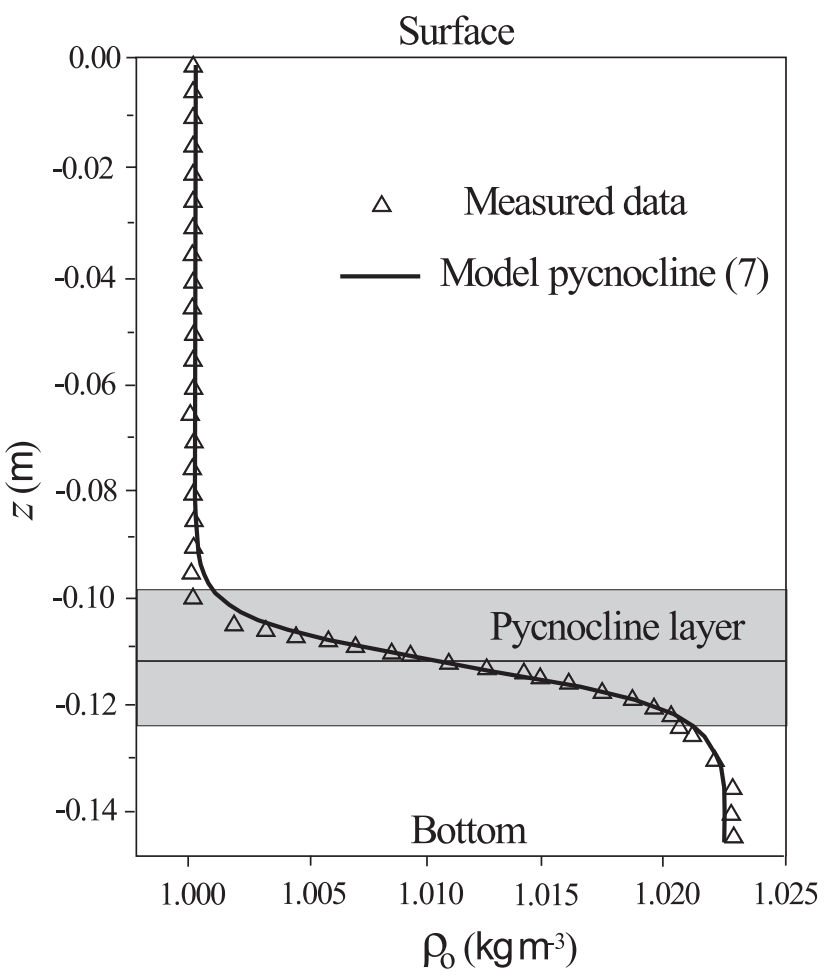

Fig. 2. Typical density profile in the channel. The measured data points $(\Delta)$ were fitted with the smooth model pycnocline (7).

part. Depending on the degree of blocking $B=H_{r} / h_{2}$, defined as the ratio of the sill height $H_{r}$ to the height of the lower layer $h_{2}$, the forward and backward moving waves either keep the solitary character or are changed into oscillating wave trains; see Diebels et al. (1994), Maurer et al. (1996). As a special case, the excitation of a second transmitted solitary wave following the first at lower speed was observed. This phenomenon is the main subject of the following description of the experiments and theoretical modeling.

There are two main parameters characterising the system, 
first the soliton amplitude and second the degree of blocking. The soliton amplitude is connected to the Froude number $F r=u_{\max } / V_{\text {lin }}$, which is defined as the maximum piston velocity of the wave generator $u_{\max }$ normalised to the linear phase speed $V_{\text {lin }}$. The range of $F_{r}$ was changed between $F_{r}=0.1$ to $F_{r}=0.8$. The best results for soliton excitation were found with $F_{r}=0.2$. This value generated an elevation amplitude of approximately $5 \mathrm{~mm}$. Higher Froude numbers produce more oscillating wave trains in the back of the solitary wave, especially when interacting with the sill. The degree of blocking $B$ was varied from 0.5 to 1 . Lower values of $B$ do not have an observable effect on the transmitted wave, whilst higher values will break down the transmitted soliton to a simple oscillating wave train. The best results splitting the incoming soliton in two parts while keeping the solitary character, were achieved for $0.7<B<0.9$.

An additional variable, the steepness of the sill (or its width $L_{r}$ ), typifying the geometry of the sill is important but does not concern the higher mode structure. As a result of the experiments, it seems to have only a small influence on the interaction with the waves. The transmitted waves are very similar to those transmitted by a short sill with the same $B$, except for their smaller amplitude.

Other parameters, such as the water densities and temperature, were kept approximately constant and are not considered any further here.

\subsection{Typical experimental data}

Figure 3 shows a typical data sheet obtained from the experiments. The elevation curves measured by the gauges are plotted versus the channel length. For each curve the same elevation scale, indicated in the upper right corner, is used, while the vertical position of the curve corresponds with the gauge position. The initial peak was approximately $5 \mathrm{~mm}$ high, decaying to about $1 \mathrm{~mm}$ height while propagating all the way down to the end of the channel. The sketch of the channel on the left of the picture explains the position of the sill and the gauges along the channel length. The dotted lines with arrows mark the path of the first mode soliton, its gradient determines the velocity which is approximately $7 \mathrm{~cm} \mathrm{~s}^{-1}$. One sees easily the splitting of the wave at the sill in the middle of the channel and the multiple reflections at both channel ends. The solid lines and arrows show the propagation of the second mode wave generated by the sill. Here the speed is only $2 \mathrm{~cm} \mathrm{~s}^{-1}$, even though the amplitude is almost equal to the transmitted first mode peak. One can indicate in Fig. 3 also a reflected second mode wave; however, owing to the smallness of the signal its existence in the experiment is questionable.

Comparison of the elevation profiles of the measured first and second mode peaks with the shape of the typical KdVsoliton which is given by the $\operatorname{sech}^{2}$-profile showed that the theoretical profiles match the experimental ones very well except in the tails where disturbances occur (Hüttemann and Hutter, 2001).

The properties of the first mode solitary wave were also

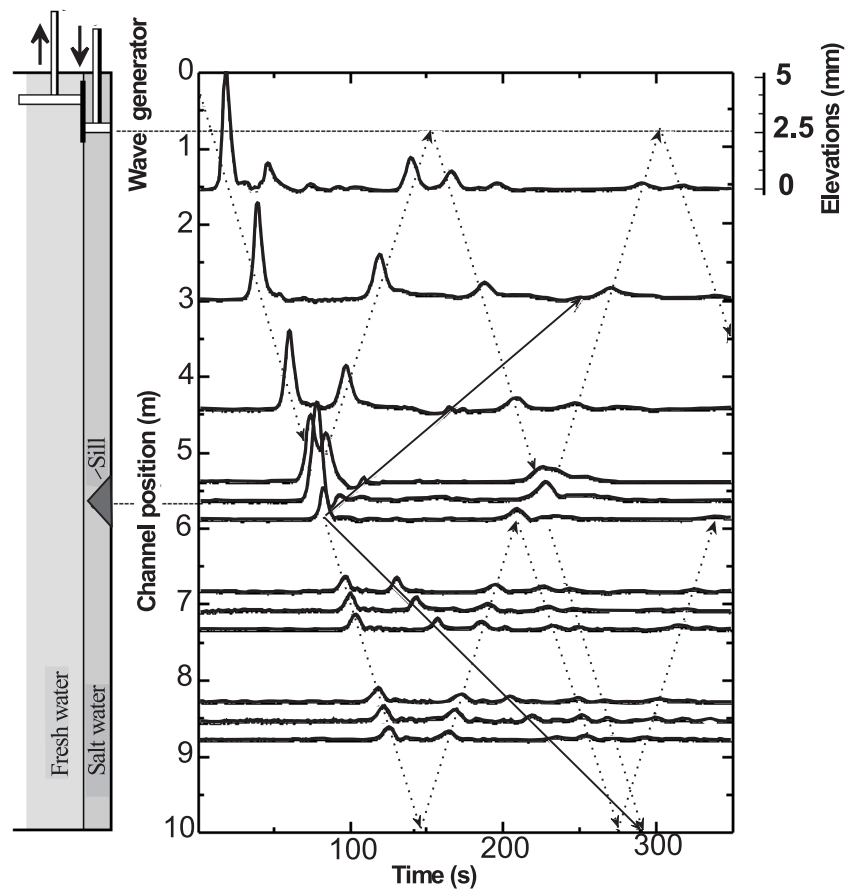

Fig. 3. Typical experimental data. Time series of the interface deflection at the six gauge positions $P 1-P 6$ (at gauges $P 4, P 5$ and $P 6$, by repeating the experiment under identical conditions, three different positions are shown). On the left, the wave channel is shown with the exact location of the sill. Dotted lines with arrows mark the propagation of the first mode, solid lines that of the second higher mode. Parameters are $F r=0.19, B=0.92$.

well discussed in (Diebels et al., 1994; Maurer et al., 1996; Wessel and Hutter, 1996) and so this paper will concentrate on the second mode peak.

The most significant difference between the two observed wave modes is their travelling speed. Theoretical estimations show that the values of the wave speed of the first two baroclinic modes are: $V_{1}=7.1 \mathrm{~cm} \mathrm{~s}^{-1}$ and $V_{2}=2.1 \mathrm{~cm} \mathrm{~s}^{-1}$. So, $V_{2}$ can be estimated to be about a third of $V_{1}$. The values of the wave speed from the experiment are: $V_{1}=7.0 \mathrm{~cm} \mathrm{~s}^{-1}$ and $V_{2}=2.0 \mathrm{~cm} \mathrm{~s}^{-1}$. The agreement between experimental and theoretical results is quite good.

Thus, the main conclusion from the experiments described above is that the single first baroclinic mode solitary wave, riding on the interface and interacting with a sill, was split by the obstacle into pairs of reflected and transmitted solitontype waves representing the first mode wave of the layered system with diffusive interface plus the second mode waves due to the finite thickness of the interface. Good agreement of the horizontal profiles of the reflected and transmitted soliton-like waves and their phase speeds of propagation with the theoretical ones permits us to make such a conclusion.

Unfortunately, however, not all the characteristics of the generated waves could be measured in the laboratory experiment. For instance, because of the use of electrical gauges instead of optical ones, the vertical and horizontal structures of the reflected and transmitted waves (velocity, density) could 
not be identified. In fact, only the analysis of the spatial structure of the solitary waves and comparison with exact analytical solutions (horizontal profiles with the $\operatorname{sech}^{2}$ - function, vertical structure with profiles of the standard boundary value problem) together with the data on the phase speed can give us the answer to the question of whether or not the observed solitary waves in the experiment are internal solitons of first or second mode.

The more successful laboratory experiments, with the generation of secondary reflected and transmitted waves of second mode, were also carried out for incident solitary waves of relatively small amplitudes ( $F r \sim 0.2$ ). Higher Froude numbers, as mentioned above, produce more oscillating wave trains in the back of the solitary waves, especially when interacting with the sill. Thus, the basic question which remains as a consequence of laboratory modeling, and which can be important for the interpretation of oceanic in-situ measurements, is as follows: what is the range of applicability of the conclusion (derived as a hypothesis) about the possibility of generation of second mode solitons during the interaction of the first mode solitons with the bottom obstacles? The theoretical model described below allows us to obtain more reliable and more substantiate conclusions in a wide range of the controlling parameters.

\section{The theoretical model}

\subsection{Model theory}

The numerical model used in the present investigation is based on the incompressible two-dimensional Navier-Stokes (NS) equations in the frame of the Boussinesq approximation. The model is capable of describing the dynamics of a continuously stratified fluid in a vertical plane. We refer the model equations to a Cartesian $x-z$ coordinate system in which the $x$-axis lies within the undisturbed free surface; the $z$-axis is vertical against the direction of gravity. In this frame, the vorticity and buoyancy equations take the forms

$\Omega_{t}+J(\Omega, \Psi)=g \frac{\rho_{x}}{\rho_{a}}+v \Delta \Omega$,

$\rho_{t}+J(\rho, \Psi)+\frac{\rho_{a}}{g} N^{2} \Psi_{x}=k \Delta \rho+k \rho_{\mathrm{ozz}}$,

where $\Omega(x, z, t)$ represents the vorticity, $\Omega=\Psi_{x x}+\Psi_{z z}$ and $\Psi(x, z, t)$ is the two-dimensional stream function

$\Psi_{z}=u, \quad \Psi_{x}=-w$,

in which $u(x, z, t)$ and $w(x, z, t)$ are the horizontal and vertical velocity components in the $x$ and $z$ directions, respectively. The reference density (which in our model is the free surface density), the density anomaly and the undisturbed density profile are denoted by $\rho_{a}, \rho(x, z, t)$ and $\rho_{0}(z)$, respectively, $N(z)=\left(-g\left(\rho_{0}\right)_{z} / \rho_{a}\right)^{1 / 2}$ is the Brunt-Väisälä frequency of the undisturbed stratification, $v$ and $k$ are the coefficients of viscosity and density diffusion and $J$ is the Jacobian operator: $J(A, B)=A_{x} B_{z}-A_{z} B_{x}$. For the calculations in this paper, the values of the eddy viscosity and diffusivity were assumed to be 0 in Sects. 4.1-4.3, where the ideal fluid is considered. In Sect. 4.4 (viscous case) the coefficients $v$ and $k$ were taken to be $10^{-6}$ and $1.4 \cdot 10^{-7} \mathrm{~m}^{2} \mathrm{~s}^{-1}$, respectively.

For the simulation of the interaction of internal solitary waves with a sill, which will be presented in the following sections, and to reproduce the conditions of the laboratory experiments presented above, the equations are numerically integrated in a domain $|x| \leq L(L=10 \mathrm{~m}),-H \leq z \leq 0$ $\left(H=0.15 \mathrm{~m}, H=h_{1}+h_{2}\right)$.

For the wave motion of the ideal fluid in Sects. 4.1-3 the following boundary conditions are imposed at the free surface $(z=0)$ and at the bottom $(z=-H)$ :

$\Psi=0, \quad \Omega=0, \quad \rho=0$.

These indicates that boundary lines are streamlines, that there are no shear stresses and that the density anomaly vanishes at the bottom. On the other hand, the influence of the bottom boundary layer, internal friction and diffusivity on the wave motions in Sect. 4.4 is accounted for by the following boundary conditions at the bottom:

$\Psi=\Psi_{x}=\Psi_{z}=0, \quad \rho_{n}=0$,

where $n$ is the normal to the bottom. Thus, the boundary line is a streamline with vanishing velocity and no buoyancy flux. The flux boundary condition for the density implies the absence of heat and salt fluxes through the bottom surface. The boundary value of the vorticity $\Omega$ at the bottom is obtained from its defining equation, $\Omega=\Delta \Psi$, where in numerical implementations the function $\Psi$ is taken from the previous temporal step.

On the vertical boundaries at the channel ends, $x= \pm L$, located sufficiently far from the origin $x=0$ (which coincides with the position of the sill) the wave motion is assumed to satisfy the homogeneous conditions

$\Psi=0, \quad \Omega=0, \quad \rho=0$.

Except for undisturbed conditions, these do not correspond to the conditions necessary to be satisfied at solid walls. They can be justified because of the existence of an upper bound of the velocity of the baroclinic disturbances, described by the system (1), (2). In our case this upper bound is the phase speed of the incident internal solitary wave. All secondary generated waves have smaller amplitudes and smaller phase velocities. The trick is to set the model boundaries sufficiently distant from the sill. Then, conditions (5) are valid so long as the leading reflected and transmitted waves have not reached the boundaries.

Equations (1), (2) with the boundary conditions (3)-(5) are solved numerically. However, before the application of the numerical scheme, system (1), (2) is transformed by means of the transformation

$x_{1}=x, \quad z_{1}=z / H(x)$.

This substitution transforms the irregular model region into a rectangular computational domain. 
The splitting-up method was used for the finite-difference approximation of the equations. At each time step, the implicit tri-diagonal matrix system is solved using standard techniques. The stream function at each time step is computed by solving the vorticity equation $\Omega=\Psi_{x x}+\Psi_{z z}$. The splitting-up method used is discussed in detail in Marchuk (1974).

The temporal spacing $\Delta t$ must satisfy the Courant-Friedrichs-Levy condition: $\Delta t / \Delta x<1 / V_{m}$. Here, $\Delta x$ is the step along the $x$-axis and $V_{m}$ is an upper bound of the phase velocity for internal waves. The majority of the calculations were carried out on a $5000 \times 100$ grid with spatial steps $\Delta x=4$ $\mathrm{cm}$ and $\Delta z=0.15 \mathrm{~cm}$.

\subsection{Model setup}

The density distribution in the numerical runs was set by choosing the following three-parameter family of curves for the Brunt-Väisälä frequency:

$N(z)=N_{\max }\left(\left[\frac{2\left(z+H_{p}\right)}{\Delta H_{p}}\right]^{2}+1\right)^{-1}$.

$H_{p}$ represents the depth where the Brunt-Väisälä frequency has its maximum $N_{\max }$ and $\Delta H_{p}$ is a vertical length scale characterising the variation of the Brunt-Väisälä frequency. This law of fluid stratification describes the experimental density profile very well (Fig. 2) with parameters $N_{\max }=3.95 \mathrm{~s}^{-1}, H_{p}=0.112 \mathrm{~m}$ and $\Delta H_{p}=0.02 \mathrm{~m}$.

The advantage of this law of fluid stratification, in comparison to piecewise linear or sigmoidal density profiles which are often used in theory and in practice for the description of pycnocline spreading (Kraus, 1966), is that it allows us to find a simple analytical solution of the standard boundary value problem for the linearized vertical baroclinic modes:

$\left(W_{n}\right)_{z z}+\mu_{n} N^{2} W_{n}=0, \quad W_{n}(0)=W_{n}(-H)=0$.

The parameters $\mu_{n}$ and $W_{n}(z)$ are the eigenvalues and the eigenfunctions of the boundary value problem, $n$ is the mode number. For the stratification (7) the solution of (8) has the simple square root trigonometric form (Vlasenko, 1994)

$W_{n}(z)=\sqrt{c_{1}\left(z / H+c_{2}\right)^{2}+1} \sin \left(n \pi z_{2}\right)$,

$\mu_{n}=(n \pi / h)^{2}-c_{1}$,

where $c_{1}=\left(2 H / \Delta H_{p}\right)^{2}, \quad c_{2}=H_{p} / H$,

$z_{2}=\left[\arctan \sqrt{c_{1}}\left(z / H+c_{2}\right)-\arctan \sqrt{c_{1}}\right] / \sqrt{c_{1}} h$,

and $h=\left[\arctan \sqrt{c_{1}}\left(c_{2}-1\right)-\arctan \sqrt{c_{1}}\right] / c_{1}$.

Let us discuss the initial conditions. The model was initialized by using the first-mode $(n=1)$ analytical solitary wave solution of the stratified KdV equation. For the stratification (7), on the basis of the remarks above, it has the very simple form (see, e.g., Vlasenko 1994):

$\Psi_{n}(x, z, t)=-a V_{n} \operatorname{sech}^{2}\left(\frac{x-V_{n} t}{\lambda_{n}}\right) W_{n}(z)$.
Here, $W_{n}(z)$ is defined by (9) and $a$ represents the wave amplitude (i.e., the maximum isopycnal displacement of the solitary wave), $V_{n}$ is its phase speed and $\lambda_{n}$ its horizontal length scale

$V_{n}=\frac{N_{\max } H}{\sqrt{\mu}_{n}}\left(1-\frac{a \gamma}{3 \delta H \mu_{n}}\right), \quad \lambda_{n}=\sqrt{-\frac{6 H^{3}}{a \gamma}}$.

Here $\delta$ and $\gamma$ are the coefficients of the Korteveg-de Vries equation,

$\delta=\int_{-H}^{0} W_{n}^{2} N^{2} d z / \int_{-H}^{0} W_{n}^{2} d z$

$\gamma=-\mu_{n} \int_{-H}^{0} W_{n}^{3}\left(N^{2}\right)_{z} d z / \int_{-H}^{0} W_{n}^{2} d z$.

For the stratification (7), these coefficients are expressed by the simple trigonometric expressions

$\gamma=\frac{96 H^{2}(n \pi)^{3}[\sin N-\sin (M+N+n \pi)]}{\Delta H_{p}^{2} M\left[M^{2}-(3 n \pi)^{2}\right]}$,

$\delta=M /\left(2 \sqrt{c_{1}}\right), \quad N=\arctan \sqrt{c_{1}}$,

$M=\arctan \sqrt{c_{1}}\left(c_{2}-1\right)-N$.

Inserting (11) in the Poisson equation for the vorticity, $\Omega=\Psi_{x x}+\Psi_{z z}$, the vorticity associated with the analytical internal solitary wave is obtained. For the internal waves of permanent form, moving in an ideal fluid with phase velocity $V_{n}$, the density equation (2) can be written as $\rho=$ $\rho\left(\Psi-V_{n} z\right)$ (Long, 1953). Imposing such a condition of isopycnic motion, the density anomaly is calculated from the undisturbed density profile. The initial fields described above represent a stationary solitary wave solution in a weakly nonlinear, weakly non-hydrostatic medium. That is why they do not satisfy the system (1), (2) in the case of large amplitude solitary waves. Once inserted in system (1), (2), they will evolve in a basin of constant depth. During the evolutional process, the initial large amplitude K-dV soliton is modified and a new stationary solitary wave is formed at the frontal side of a wave field. The leading wave, having the larger phase speed in comparison with that of the wave tail, separates from the latter at a definite stage of evolution (at the distance of $20 \div 30$ wavelengths from its incept) and propagates further independently as a solitary wave. Thus, the model is run until a leading wave separates from a wave tail and until a new stationary solution is reached at the frontal side of the wave field. This wave is then used as initial condition for the problem of the interaction of solitary waves with a sill.

We ought to dwell briefly upon the properties of such initial solitary waves of finite amplitude and their differences from K-dV solitons described by (11). Detailed theoretical and experimental analyses of specific features of large amplitude solitary internal waves for a two-layered fluid were presented, for instance, in papers Miyata $(1985,1988)$ and Michallet and Barthelemy (1998), respectively. Their extensions to a continuously stratified fluid are contained in 

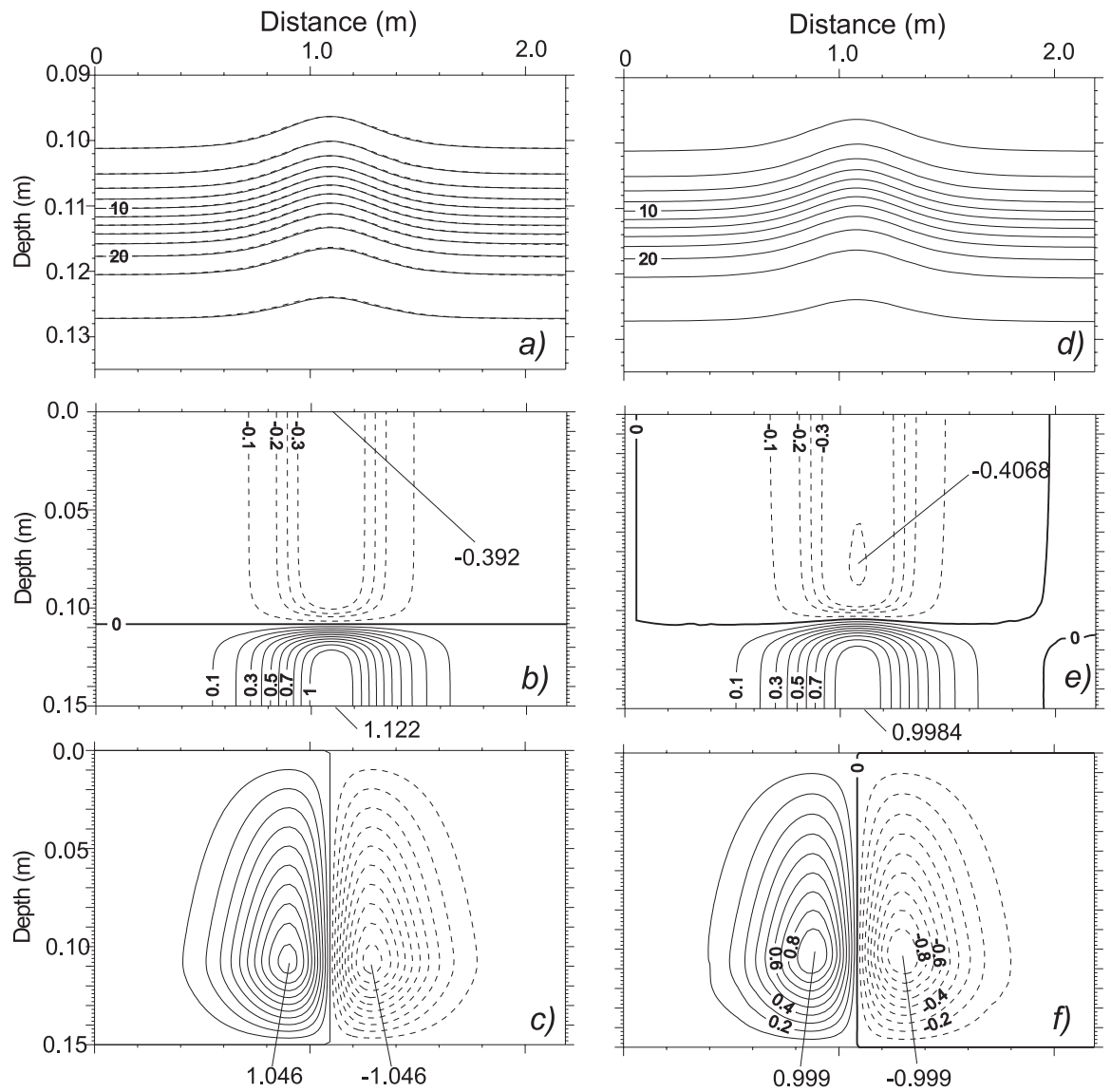

Fig. 4. Initial solitary internal wave. The left column represents the initial analytical K-dV soliton with amplitude $a=5 \mathrm{~mm}(F r=0.13)$, the right column displays the numerical results of Eqs. (1), (2) after an integration in time from the initial $\mathrm{K}-\mathrm{dV}$ state of duration $t=50 T\left(T=\lambda_{1} / V_{1}\right)$ over a flat bottom. Panels (a) and (d) show the density anomaly relative to the free surface $\left(\mathrm{kg} \mathrm{m}^{-3}\right)$, panels $(\mathbf{b})$ and $(\mathbf{e})$ show the fields of horizontal velocity $u\left(\mathrm{~cm} \mathrm{~s}^{-1}\right)$ (c) and (f) those of the vertical velocity $w\left(\mathrm{~mm} \mathrm{~s}^{-1}\right)$. For comparison the numerical solitary wave is also presented in Fig. 4a by dashed lines.
Lamb and Yan (1996) and Vlasenko et al. (2000). One result of these papers is that the real, observed internal solitary waves of large amplitude have several specific features in their structure which increase with the growth of soliton amplitude. For instance, they are wider and propagate more slowly than the K-dV solitons. Furthermore, their vertical structure does not coincide exactly with the eigenfunctions (9) of the linear boundary value problem (8). And, finally, the larger the amplitudes, the larger is the difference between the real solitary waves and the K-dV solitons. Nevertheless, relatively small solitary waves can be successfully described by the weakly nonlinear theory.

Figures 4, 5 display a comparison of the initial K-dV internal soliton $(a=5 \mathrm{~cm})$ with its numerical counterpart of the same amplitude. The first soliton is the solution of the weakly nonlinear theory, the second is the numerical solution of the full system (1), (2), defined as a result of the adjustment of the $\mathrm{K}-\mathrm{dV}$ soliton to the ambient conditions. In the present case, i.e., for small Froude number $(F r=0.13)$, the difference between the two is rather small but can nevertheless be seen in the vertical profiles of isopycnal displacement and the vertical and horizontal velocity components; it increases with increasing amplitude. More on this will be presented below and can also be found in the mentioned literature.

\section{Results of the numerical experiments}

Numerical experiments were carried out to reproduce the laboratory experiments described above. The mechanism of interaction of the internal solitary wave with an obstacle is discussed in detail on the basis of one numerical experiment (basic case run). All input parameters of the model (stratification, configuration of the sill, amplitude of the incident wave) in this run (Sect. 4.1) are very close to those in the laboratory experiment. To eliminate the influence of viscosity and diffusivity in the first stage of investigation the coefficients $v$ and $k$ were taken as small as possible to guarantee numerical stability of the scheme $\left(10^{3}\right.$ times smaller than those in the laboratory modelling). The influence of the viscosity and diffusivity on the results will be discussed in Sect. 4.4. The sensitivity of the model results to the height of the sill and to the amplitude of the incident wave will be discussed in Sects. 4.2 and 4.3 .

\subsection{Basic case run}

We will use the background fluid stratification (7), defined in Sect. 3.2, throughout. To reproduce the laboratory experiment, the amplitude of the incident wave was taken to be 5 $\mathrm{mm}$, corresponding to the laboratory Froude number 0.13 . In the numerical runs, the Froude number was defined as the ratio of the maximum horizontal orbital velocity $u$ (localized at 

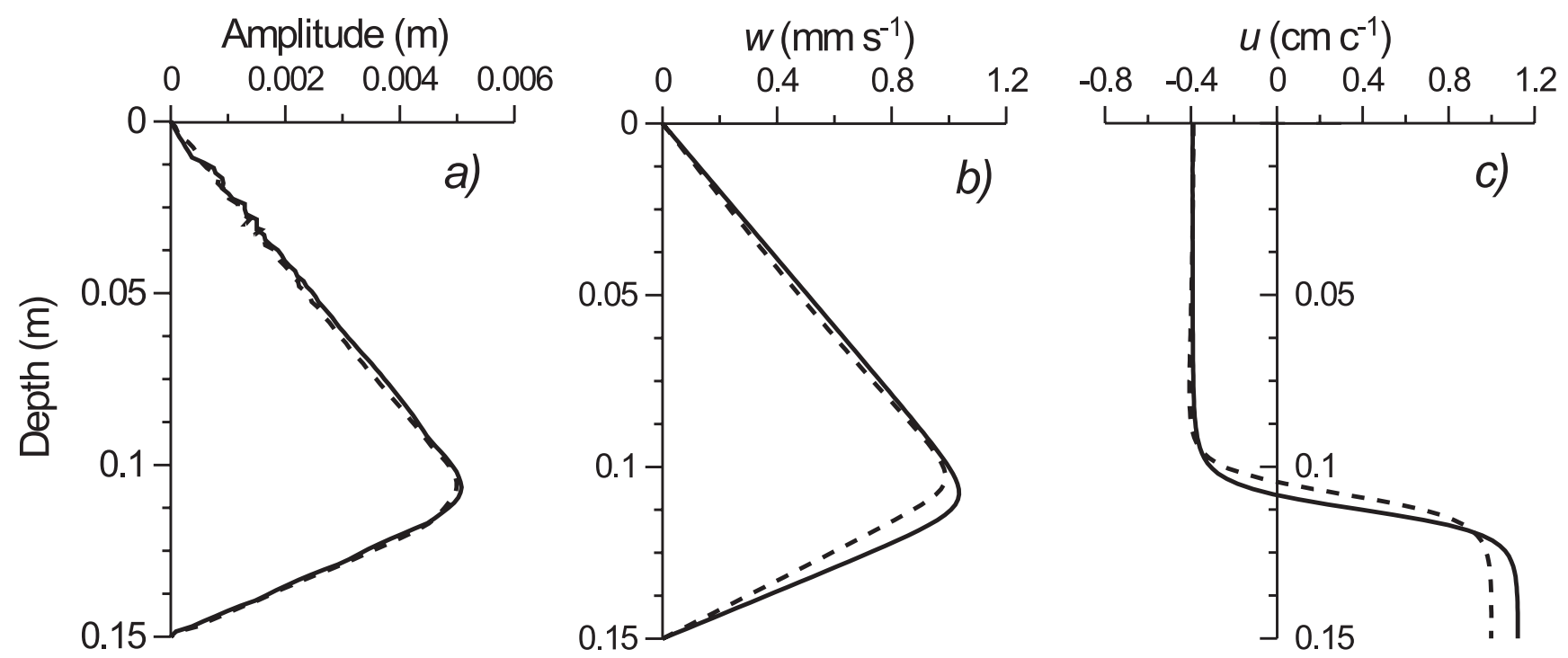

Fig. 5. Vertical profiles of the initial K-dV soliton (solid lines) $(a=5 \mathrm{~mm}, F r=0.13)$ compared with the numerical solitary wave (dashed lines) of the same amplitude (after a time $t=50 T$ from initial onset, see also Fig. 4 caption). Isopycnal displacements (a) and horizontal velocities (c) calculated at the wave centre, vertical velocities (b) calculated at the position of maximum vertical velocity.

the bottom) in the incident wave to the linear phase speed $V_{1}$ defined by the boundary value problem (8). In the laboratory experiment, the Froude number was defined using the maximum piston velocity. This value is not exactly equal to the maximum of the horizontal orbital velocity of fluid particles in the generated solitary wave because a non-negligible part of the energy supplied by the piston was spent for the generation of secondary waves. In any case, the conformity of the numerical runs with the laboratory experiments was maintained by choosing the same amplitude of the incident wave, which in the laboratory experiments was measured very accurately. The height of the sill $H_{r}$ was equal to $3.2 \mathrm{~cm}$ (blocking parameter $B$ equals to 0.92 ), and its length $L_{r}$ was $60 \mathrm{~cm}$, as in the laboratory experiments described in Sect. 2.3.

The vertical and horizontal structures of the incident wave were discussed in Sect. 3.2. Now consider its evolution in a basin of variable depth. Figure 6 shows the process of interaction of the solitary wave with a triangular sill. Snapshots of isopycnal lines are presented for four consecutive times. The time scale $T$ is defined as $T=\lambda_{1} / V_{1}$, where $V_{1}$ is the nonlinear phase velocity of the Korteveg-de Vries soliton having an amplitude of $0.5 \mathrm{~cm}$, and $\lambda_{1}$ is its length, see Eq. (12). Only the more representative time slices are shown. The initial wave motion is from right to left. The beginning of interaction occurs at $t=5 T$ (Fig. 6a), the passage of the wave behind the sill at $t=6 T$ (Fig. 6b), the formation of the first and second transmitted and reflected waves at $t=9 T$ (Fig. 6c) and the final stage of evolution at $t=17 T$ (Fig. 6d) with fully established transmitted and reflected solitary waves. The directions of propagation of all waves are indicated in panel (d) by arrows.

The first transmitted and first reflected waves look qualitatively like the first mode solitary waves with co-phase dis- placements of isopycnals through the entire depth (from the surface to the bottom). At the same time, the more intensive second transmitted and reflected waves are characterised by the counter-phase displacements of the isopycnals below and above the centre of the pycnocline. This qualitatively coincides with the behaviour of the solution of the standard boundary value problem for internal waves, if we take into account the "rigid lid" condition (3) at the free surface. In this case the first and second eigenfunctions (9) have at depth only one or two extrema, respectively. Thus, in the numerical run the incident solitary wave is split over the sill into two pairs of reflected and transmitted solitons of the first and second mode. This conclusion is drawn from purely qualitative considerations of Fig. 6, but only careful analysis of the horizontal and vertical structure of such waves, their kinematic characteristics (phase speed, length scale) and comparison with the analytical solution (K-dV soliton in the present case) can provide the devinitive answer to the question, whether these waves are really internal solitons of the first and second mode.

To check this hypothesis let us analyse the detailed structure of the waves and compare them with the K-dV solitons of the same amplitude. This comparison is performed for the time $t=35 T$.

Figure 7 shows the structure of the first transmitted wave. The vertical profiles of $u$ and $w$ are presented as well as the isopycnal lines, the horizontal and vertical velocities. The former are built at places where the maximum of $u$ or $w$ occurs. For comparison, the corresponding profiles of the K-dV soliton, having the same amplitude, are presented by dashed lines.

It can be seen that the analytical K-dV soliton describes the first transmitted wave rather well. The reason for the 


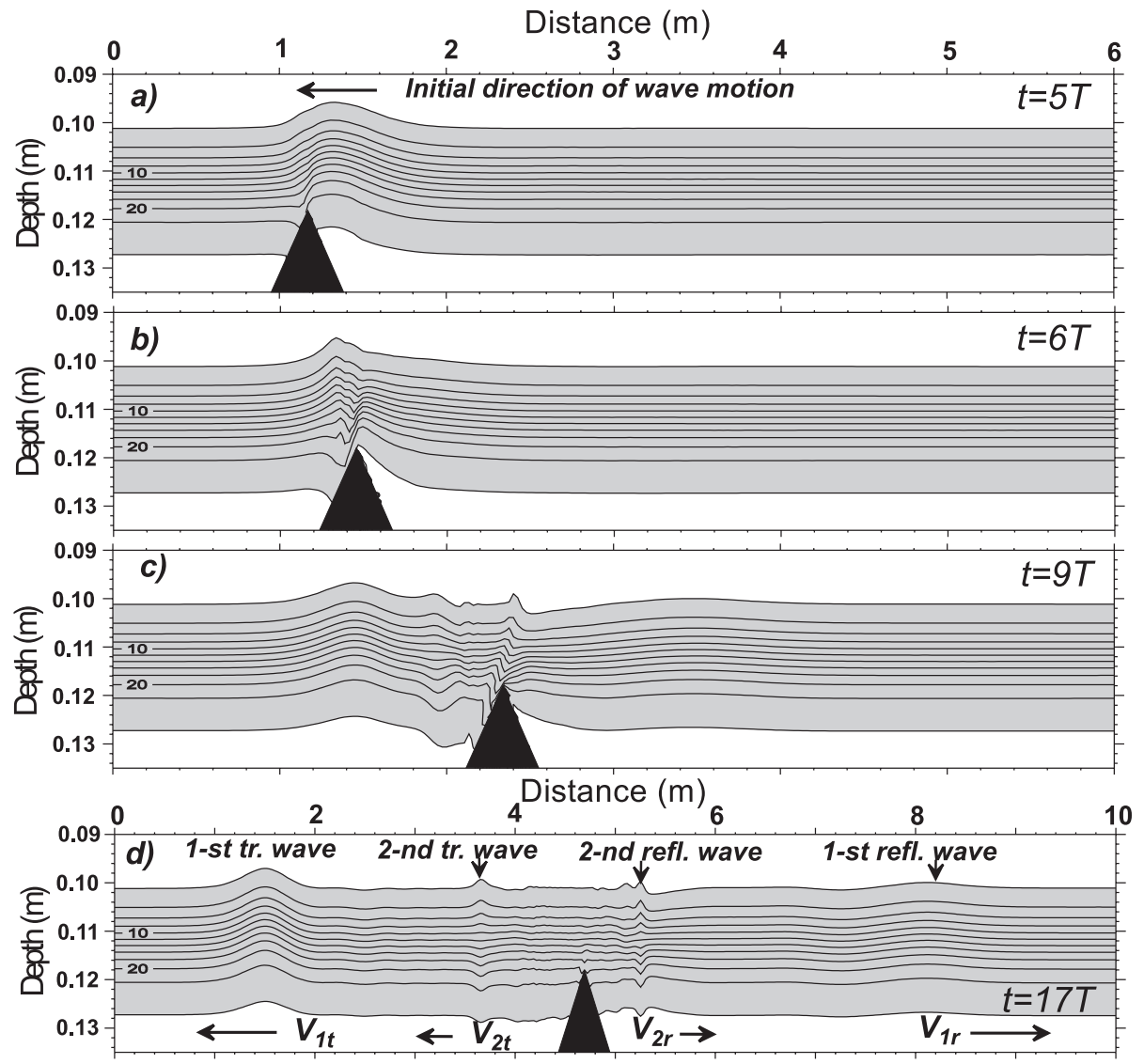

Fig. 6. Evolution of the density field (density anomaly relative to free surface $\left(\mathrm{kg} \mathrm{m}^{-3}\right)$ ) during the interaction of a first mode solitary wave with the sill. First and second reflected and transmitted waves are presented in the lower panel at $t=17 T$. nonessential discrepancy of the analytical and numerical profiles was discussed in Sect. 3.2; it is connected with the difference of the real finite amplitude solitary waves and the analytical soliton defined within the frame of weaklynonlinear theories. For the wave with amplitude of $3.86 \mathrm{~mm}$ $(F r=0.102)$ this difference is not very large anyway. Moreover, the phase speed of its propagation equals $0.996 V_{1}$. So one can conclude that the characteristics of the first transmitted solitary wave are very close to the analytical ones and this wave can be considered almost as a K-dV soliton.

The same conclusion can be reached for the first reflected wave. This has an amplitude of $1.28 \mathrm{~mm}(\mathrm{Fr}=0.032)$ and is presented in Fig. 8. A somewhat less convincing coincidence of this wave with the $\mathrm{KdV}$ soliton can be explained by the relatively short distance of propagation from the sill (approximately 20 units of wavelength). This wave has a 3 times smaller amplitude than the first transmitted wave and, consequently, it is wider; its wavelength equals $51.6 \mathrm{~cm}$ as compared to $32.5 \mathrm{~cm}$ for the first transmitted wave but its phase speed differs from the linear speed (speed of propagation of a wave tail) only by $0.5 \%$ as compared to $2.5 \%$ for the first transmitted wave. So the first reflected wave must travel an additional distance of several tens of wavelengths for separation from the tail. The latter is clearly seen in Fig. 8. Nevertheless, one can conclude that somewhere far from the sill its characteristics will be very close to the theoretical K-dV soliton.

Let us now consider the structure of the second transmitted wave which was treated in the laboratory experiment as a second mode soliton. Its structure is shown in Fig. 9 and compared with the second mode analytical K-dV soliton of the same amplitude ( $a=1.47 \mathrm{~mm}$ ) (dashed lines for $u$ and $w$ profiles). Agreement of the numerical and analytical curves is fair. The discrepancy can be explained by the fact that the second transmitted wave does not propagate into an undisturbed medium but into the background of the wave tail remaining behind the first transmitted first mode soliton.

The second reflected wave (Fig. 10) shows completely different characteristics. In fact, this signal can not be considered as a single solitary wave but only as a wave train. It consists of three consecutive waves arranged in sequence according to the magnitude of their amplitudes. In the front part of the train the waves possess the structure of the second baroclinic mode with a counterphase displacement of the isopycnals above and below the pycnocline centre but, in the tail, one can also find manifestations of the third and fourth baroclinic mode. Moreover, comparison of such waves with the 2nd mode $\mathrm{K}-\mathrm{dV}$ solitons of the same amplitude shows that the analytical wave is several times wider and, as a consequence, its vertical velocity is almost three times smaller. The last feature of the numerical waves is that they "live" on the pycnocline. Their horizontal velocity attenuates expo- 

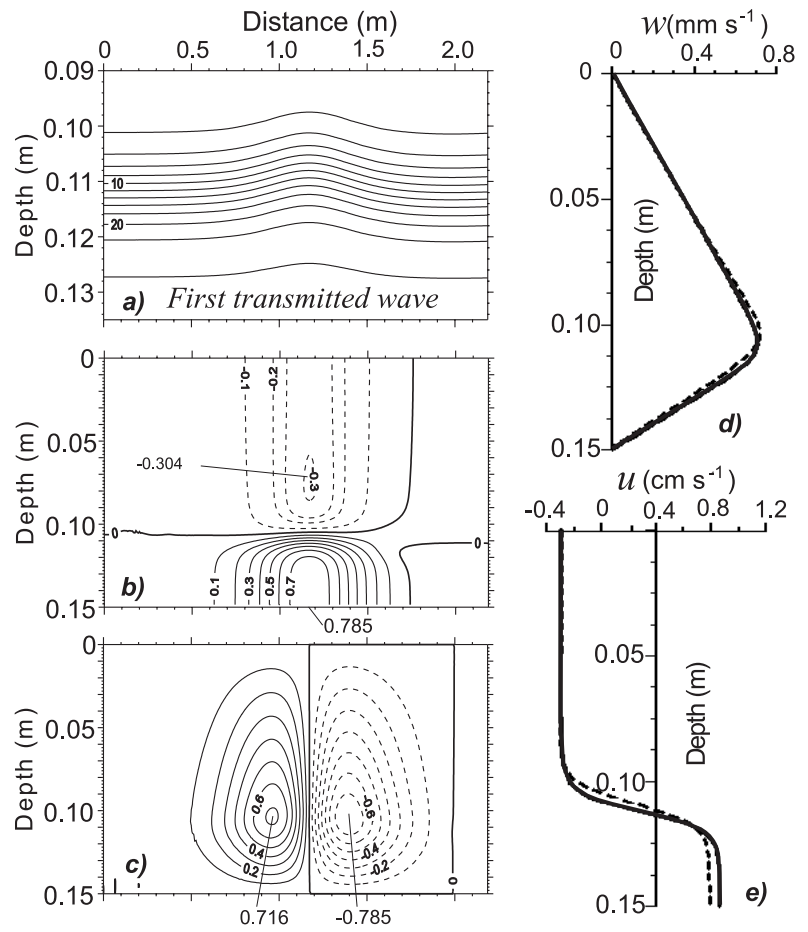

Fig. 7. (a) Density field $\left(\mathrm{kg} \mathrm{m}^{-3}\right)$, (b) horizontal $\left(\mathrm{cm} \mathrm{s}^{-1}\right)$ and (c) vertical $\left(\mathrm{mm} \mathrm{s}^{-1}\right)$ velocity fields of the first transmitted wave. On the right the vertical profiles of the horizontal (e) and vertical (d) velocities are presented by dashed lines. For comparison, the vertical profiles of the K-dV soliton of the same amplitude are shown by solid lines.
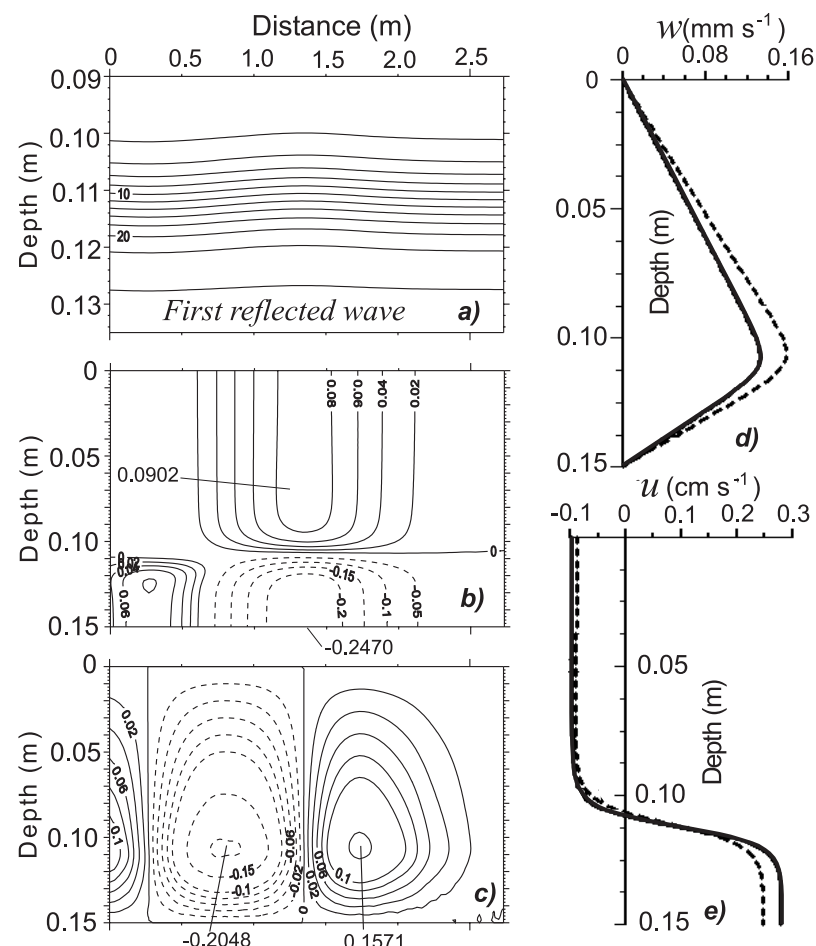

Fig. 8. The first reflected wave. Notations in this figure are the same as in Fig. 7.
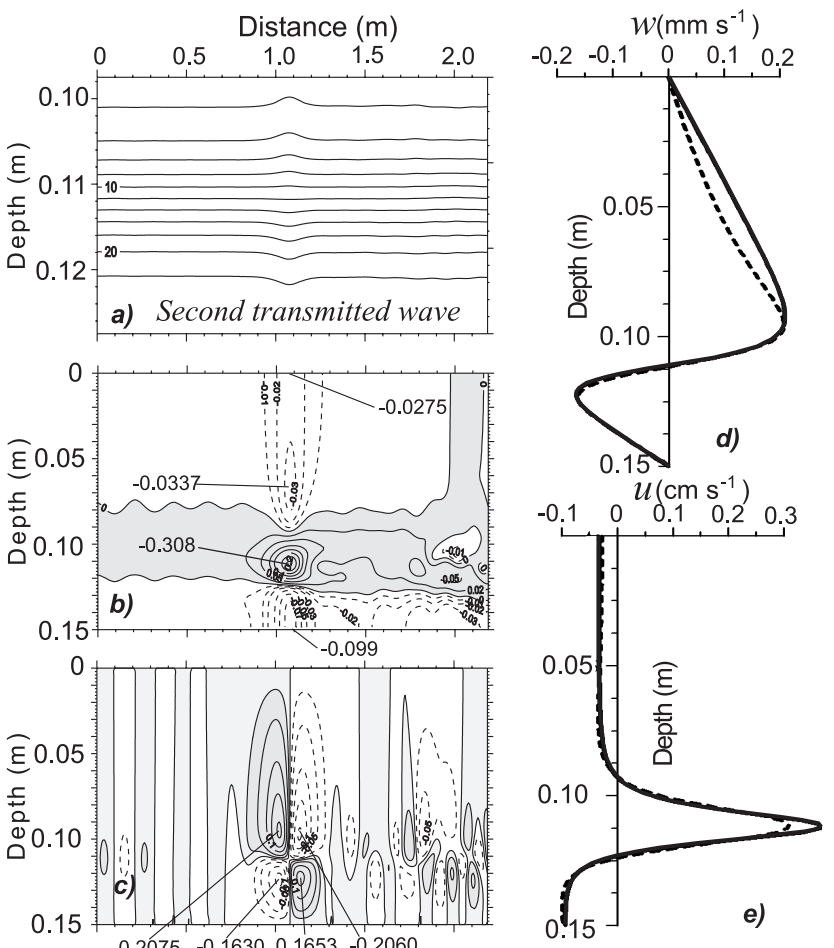

Fig. 9. The second transmitted wave. Notations in this figure are the same as in Fig. 7.
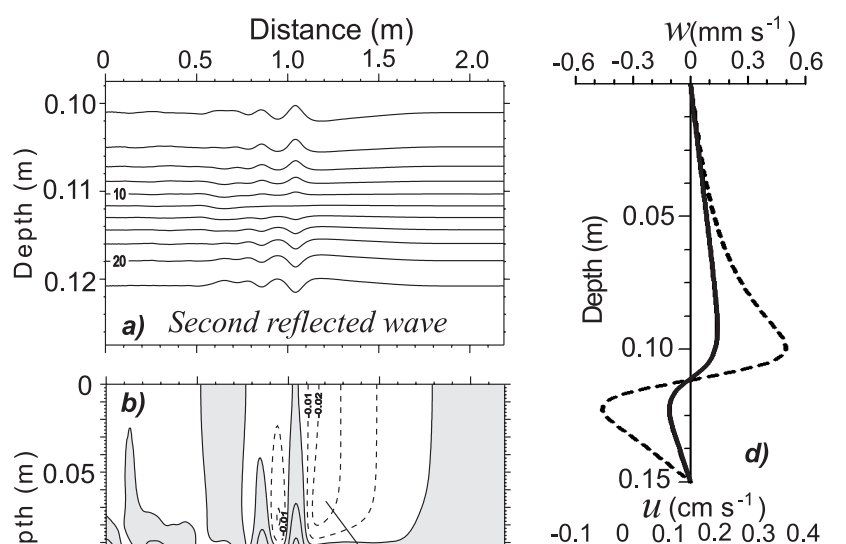

$\begin{array}{lllllllllllll}-0.1 & 0 & 0.1 & 0.2 & 0.3 & 0.4\end{array}$
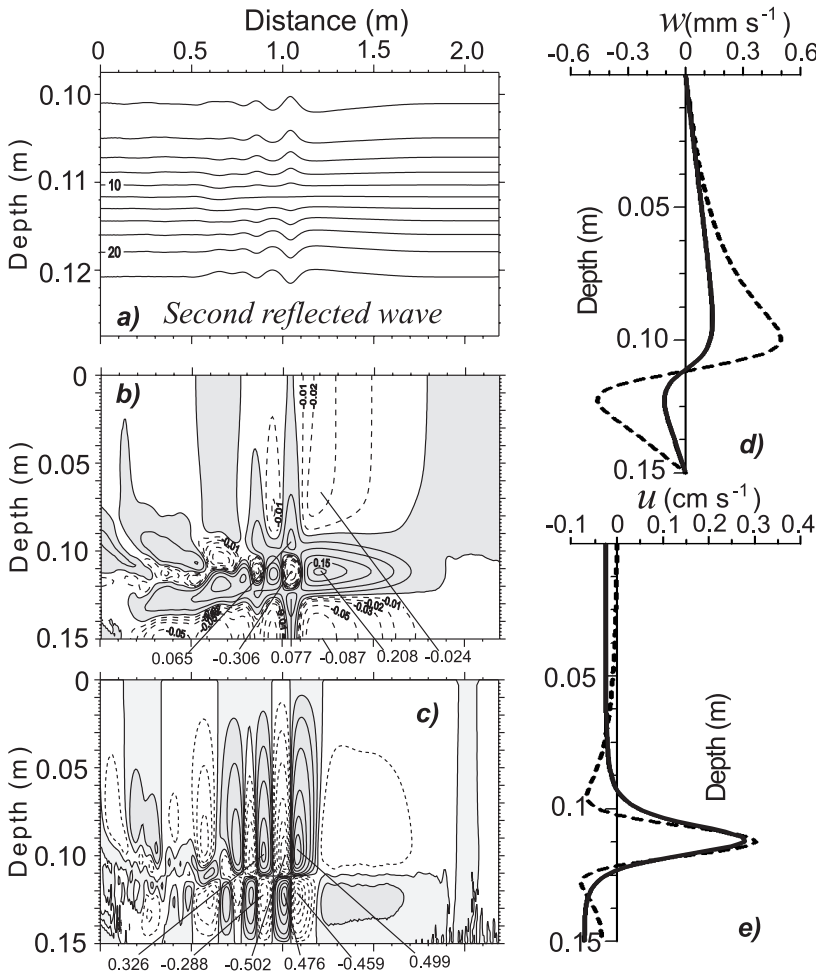

Fig. 10. The second reflected wave. Notations in this figure are the same as in Fig. 7. 
nentially in directions away from the pycnocline to the free surface and to the bottom. Thus, our analysis shows that the second reflected wave signal can not be treated as a solitary wave of the second baroclinic mode.

\subsection{Dependence on sill height (blocking parameter $B$ )}

The results of comparison runs are presented in this section. The runs were performed in order to evaluate the influence of the height of a sill on the characteristics of the reflected and transmitted waves. In experimental work (Wessels and Hutter, 1996) the main question under study was to delineate the portions of an incoming wave that are reflected, transmitted or dissipated. It was found there that, for a two-layered fluid, the blocking parameter $B$ is the basic parameter controlling the transfer of the energy across an obstacle. One would expect that in our case of smoothed pycnocline this parameter does not only control the amount of transmitted and reflected energy but also the redistribution (splitting) of the energy between first and higher baroclinic modes. So, contrary to the paper of Wessels and Hutter (1996), we now formulate a wider question and try to recognize what might be the influence of the blocking parameter on the quantitative characteristics (amplitudes) and on the qualitative structure (comparison with $\mathrm{K}-\mathrm{dV}$ solitons) of the reflected and transmitted waves.

Figure 11 displays the dependence of the amplitudes of the reflected and transmitted (first and second) waves on the sill height. These are defined as the maximum displacements of the isopycnals from the state of equilibrium. The figure indicates a decrease of the amplitude of the first transmitted wave (the incoming wave looses part of its energy) and an increase of the amplitudes of both reflected and second transmitted waves with an increase of $B$. This demonstrates a growing effective energy transfer with increasing blocking parameter from the incoming first mode solitary waves to the secondary scattered waves. As in the experimental work of Wessels and Hutter (1996), the scattering of the energy of the incoming wave may be ignored, however, when $B<0.6$.

The next interesting feature is that all secondary generated waves (both reflected and second transmitted) have almost equal amplitudes in the entire considered range of $B$ values. This is, probably, connected with the fact that these waves are generated by a single wave disturbance which arises over the sill during its interaction with the incident wave (see, for instance, Fig. 6 at $t=6 T$ ). After some time it splits due to the dispersion into first and second baroclinic wave disturbances.

The reflected and transmitted waves for the basic case run were compared with the analytical solution (11). Let us analyse how the conclusion obtained from that study about the soliton character of the first reflected and both transmitted waves depends on the height of the obstacle. As in Sect. 4.1, we compare all solitary waves with the K-dV solitons having the same amplitude. Figure 12 summarizes this comparison. All curves here are normalised to the maximum value of the appropriate K-dV soliton. This figure suggests that first

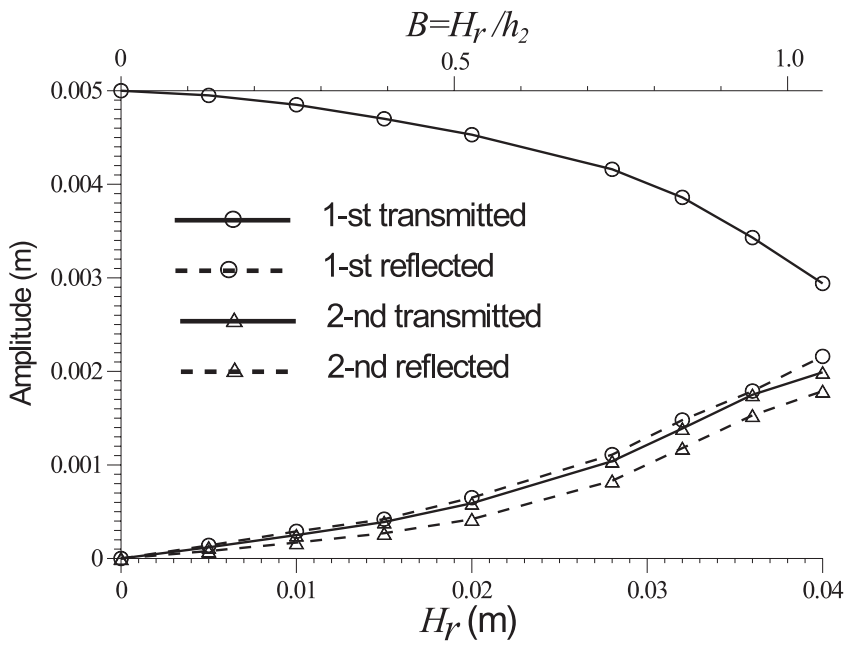

Fig. 11. Amplitudes of transmitted and reflected waves plotted against the degree of blocking $B=H_{r} / h_{2}$. Notations are shown in the figure.

and second transmitted waves fit the analytical solution (11) quite well over a wide range of the parameter $B(0<B<1)$. Large discrepancies between numerical and analytical curves for the second transmitted waves can be explained by the fact that these waves do not propagate in the undisturbed fluid but on the background of the weak first mode wave train and thus remain in the tail of the first transmitted wave. The existence of such a dispersive wave train was also indicated in Wessels and Hutter (1996).

The behaviour of the vertical profiles of the first and second reflected waves corroborates the conclusion, formulated in Sect. 4.1, that the first reflected wave can be interpreted as the first mode $\mathrm{K}-\mathrm{dV}$ soliton type wave but that the second one belongs to a second mode dispersive wave train whose characteristics are very far from a second mode K-dV soliton (they are essentially shorter).

As a rule, the maximum of the vertical velocity of the computed first reflected wave is somewhat larger than that of the analytical solution, probably because the first reflected waves are shorter than those of the analytical solitons. To check this idea the integral lengthscale (hereafter called "wavelength") defined as

$\lambda(z)=\frac{1}{2 a(z)} \int_{-\infty}^{\infty} \zeta(x, z) d x$

was estimated for every wave. Here $\zeta(x, z)$ represents the displacement of the isopycnal whose undisturbed depth is $z$, and $a(z)$ is its value at the wave centre. The defined wavelength, when applied to the K-dV soliton (11), gives the value defined by formula (12).

Figure 13 shows the wavelengths of all computed reflected and transmitted first and second solitary waves compared to theoretical values defined by formula (12). Reflected first mode solitary waves are represented here by triangles. Evidently weak reflected waves, having amplitudes less than 


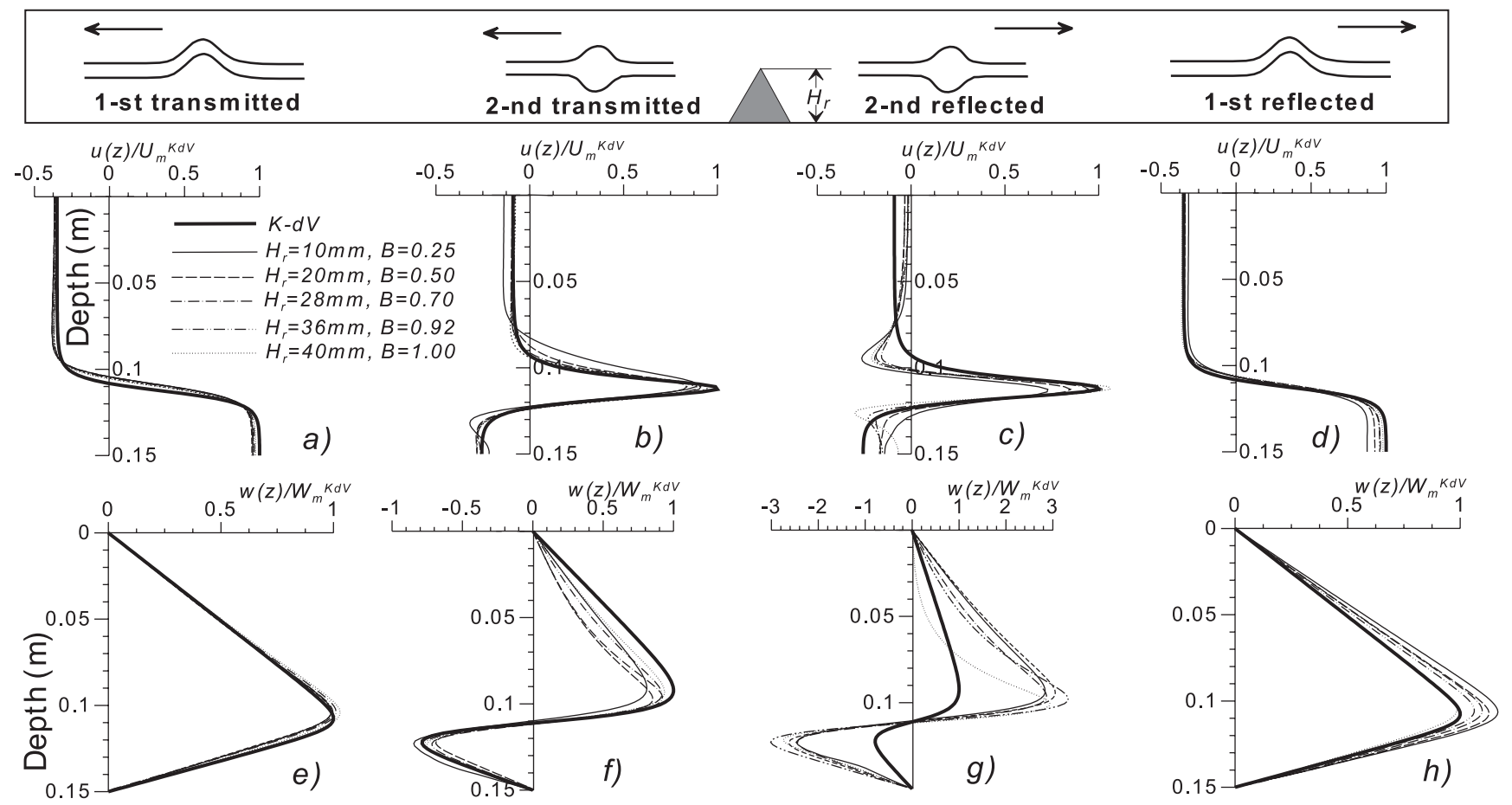

Fig. 12. Vertical profiles of horizontal (a)-(d) and vertical (e)-(h) velocities of transmitted and reflected waves plotted for several values of the blocking parameter $B$. Profiles were normalized to the maximum values of the appropriate K-dV solitons having the same amplitude. First reflected and first transmitted waves are compared with the first mode K-dV solitons, second reflected and second transmitted waves with the second mode KdV solitons. Normalised profiles of the K-dV solitary waves are presented by thick solid lines. All other notations are shown in the figure.

$0.2 \mathrm{~cm}$, are shorter than the analytical K-dV solitons. This can probably be explained by a relatively small distance of propagation from the source of generation (compared with its wavelength).

In summary, the blocking parameter (the height of the sill) controls basically the energy loss of the incoming wave and its transfer to reflected and transmitted secondary waves but it has a weak influence on the modal and spatial structure of the wave field.

4.3 Dependence on the amplitude of the incoming wave (Froude number $\mathrm{Fr}$ )

Next we focus attention on the sensitivity of the results to the amplitude of the incoming wave. The analysis of the structure of the reflected and transmitted waves is also carried out with the use of the analytic solution (11). The K-dV theory is valid asymptotically for weak nonlinearities of the considered waves, that is solution (12) holds for $a / H \ll 1$. An increase of the amplitudes leads to a discrepancy between real internal solitary waves and the $\mathrm{K}-\mathrm{dV}$ solitons. For large-amplitude solitary waves in a two-layered fluid this discrepancy was previously theoretically established by Miyata $(1985,1988)$ and later confirmed experimentally by Michallet and Barthelemy (1998). For a continuously stratified fluid, large-amplitude internal solitary waves, out of the range of applicability of weakly nonlinear theories, were nu- merically studied by Vlasenko et al. (2000). One conclusion is that the lengthscale of large-amplitude internal solitary waves is larger and their propagation speed is lower than those of the $\mathrm{K}-\mathrm{dV}$ solitons. In contrast to the results of the K-dV model, for large amplitudes the wavelength increases with increasing amplitude. The space structure of such strong waves also has several specific features in comparison with weakly nonlinear waves. For instance, the vertical profiles of the horizontal and vertical velocities of such waves only match well with the eigenfunctions of the linear boundary value problems (8) for relatively weak waves. The depth of the maximum isopycnal displacement, depth of zero horizontal velocity and depth of maximum vertical velocity are functions of the amplitude. The difference increases with growing amplitude. This leads, for instance, to a shift of the locus of zero horizontal velocity from the pycnocline with a simultaneous decrease of the maximum of horizontal velocity. Because strong solitons are wider, the maximum of the vertical velocity $w$ for such waves is also smaller than that of the K-dV soliton.

The marked peculiarities of the large amplitude solitary waves can also be found in Fig. 13 on the series of profiles of the first transmitted waves. Profiles were normalised to the maximum value of the appropriate $\mathrm{K}-\mathrm{dV}$ soliton with the same amplitude. K-dV profiles are shown in Fig. 13 by thick lines. The amplitude of incoming waves is changing in this series from 0 to $21.7 \mathrm{~mm}$ (corresponding to the 
Amplitude of a second mode $(\mathrm{m})$

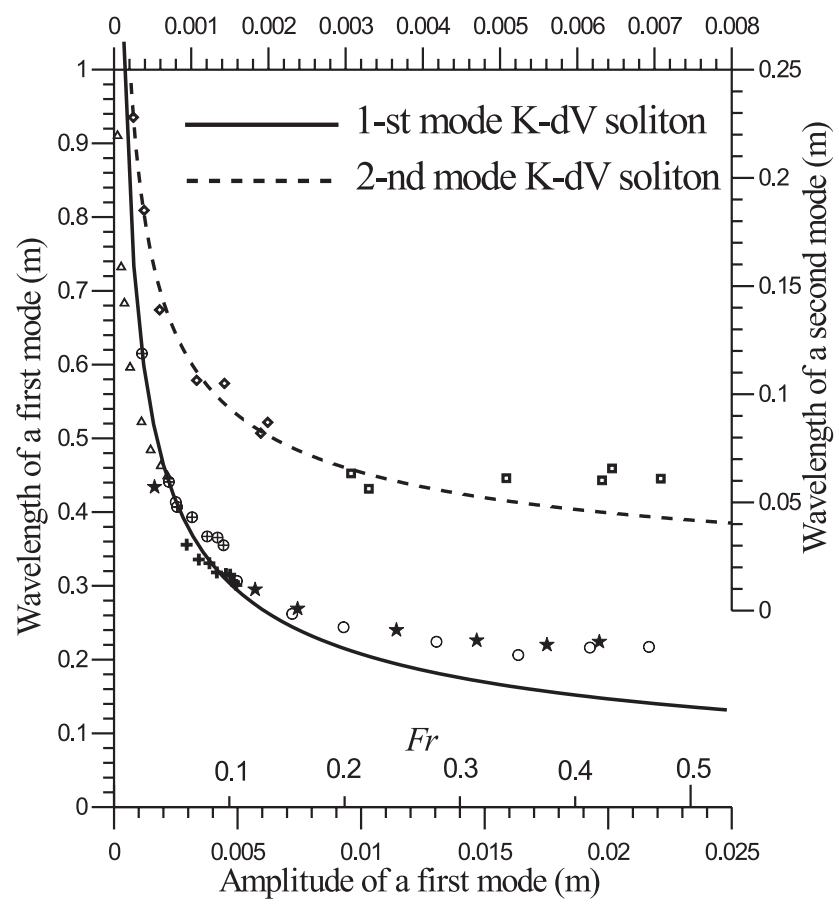

Fig. 13. Wavelengths of the solitary waves plotted against wave amplitude. Solid and dashed lines denote the theoretical curves for the first and second mode K-dV solitary waves respectively. The data points were calculated with the help of formula (13) for numerical solitary waves (incoming, reflected and transmitted). Symbol (o) denotes the incoming wave, symbol (+) - first transmitted wave obtained in Sect. 4.2, symbol $(\triangle)$ - first reflected wave obtained in Sect. 4.2, symbol $(\star)$ - first transmitted wave obtained in Sect. 4.3, symbol $(\otimes)$ - first reflected wave obtained in Sect. 4.3, symbol $(\diamond)$ second transmitted wave obtained in Sect. 4.2, symbol ( $\square)$ - second reflected wave obtained in Sect 4.3.

Froude numbers from 0 to 0.465$)$. So, this figure shows the difference between the numerical strong internal solitary and $\mathrm{K}-\mathrm{dV}$ waves. Satisfactory agreement exists between numerical and $\mathrm{K}-\mathrm{dV}$ profiles for small amplitudes; deviations occur with increasing amplitude. For small amplitudes $(F r<0.2)$, the wavelengths of all considered incident and transmitted first mode solitary waves also fit the theoretical K-dV dependence $\lambda=\lambda(a)$ with good accuracy. Deviations occur for large amplitude waves (see Fig. 13) in accordance with Miyata (1985; 1988), Michallet and Barthelemy (1998) and Vlasenko et al. (2000).

The above results are valid for incident and first transmitted waves with amplitudes such that $0<F r<0.5$. All other (reflected and second transmitted) secondary waves have substantially smaller amplitudes. This is why they can be compared with the K-dV solitons. For instance, the first reflected waves can be considered as $\mathrm{K}-\mathrm{dV}$ solitons of the first baroclinic mode (see Fig. 14).

One interesting peculiarity of the above generation of second-mode-solitary waves and its dependence on the amplitude of the incoming wave should be emphasized. In the basic case run (at $F r=0.13$ ), the second transmitted wave was treated as a second mode K-dV soliton, whereas the second reflected wave in fact was a dispersive packet of shorter waves. This situation, being valid at small Froude numbers $(F r<0.2)$, reverse with an increase of the wave amplitude. The second reflected wave at $F r=0.23$ fits the analytical K-dV profile substantially better than at $F r=0.069 ; 0.130$ (see Fig. 14). With the increase of the amplitude agreement between $\mathrm{K}-\mathrm{dV}$ theory and numerical results worsens. These profiles were built with the use of the resulting wave field which includes not only the second mode solitary wave but also the first mode wave tail left behind the first reflected wave. This fact counteracts a better agreement.

On the other hand, the dependence of the characteristics of the second transmitted waves on the Froude number is the opposite. With an increase of the amplitude of the incoming waves its energy is transferred behind the sill more effectively into the dispersive first-mode-wave train accompanying the leading solitary wave than into the second mode solitary wave (see Fig. 14). This result was mentioned in the experimental work by Wessels and Hutter (1996). The second reflected wave in this case represents an extensive bulge of a pycnocline (wider than that of the K-dV soliton).

\subsection{Influence of viscosity}

In the laboratory experiments performed for the two layered fluid (Maurer et al. 1996; Wessels and Hutter 1996), the influence of the viscosity on the amplitude and potential energy of the propagating internal solitary waves were studied in detail. It was found that the wave characteristics are sensitive to the wall friction and internal friction at the fluid interface. One result from these studies was that the wave amplitude decays exponentially with distance during the propagation of the internal wave. For instance, at a distance of $10 \mathrm{~m}(30 \div 35$ characteristic wavelengths) the amplitude of the first mode solitary wave is reduced to approximately $40 \%$ of its initial value.

These results on the attenuation of solitary waves due to the viscosity are essentially corroborated by us, because the background conditions of the numerical runs (characteristic scales of a basin, parameters of fluid stratification and excited internal waves) were the same as in the laboratory experiments.

Figure 15 shows two series of vertical profiles of first and second reflected and transmitted waves obtained for ideal and viscous fluids for the parameters $F r=0.13, B=0.92$. The essential difference lies in the vertical profiles of horizontal and vertical velocity components. The first difference is connected with the behaviour of the horizontal velocity profiles near the bottom. In the viscous case the bottom boundary layer can readily be seen. The energy loss in this layer and inside a pycnocline, due to the presence of the vertical shear stresses, leads to a decrease of the wave amplitude. Estimation of this decay shows that the amplitude of the horizontal velocity, in the presence of a bottom boundary layer, decreased by $45 \%$ for the first transmitted and by $25 \%$ for first 


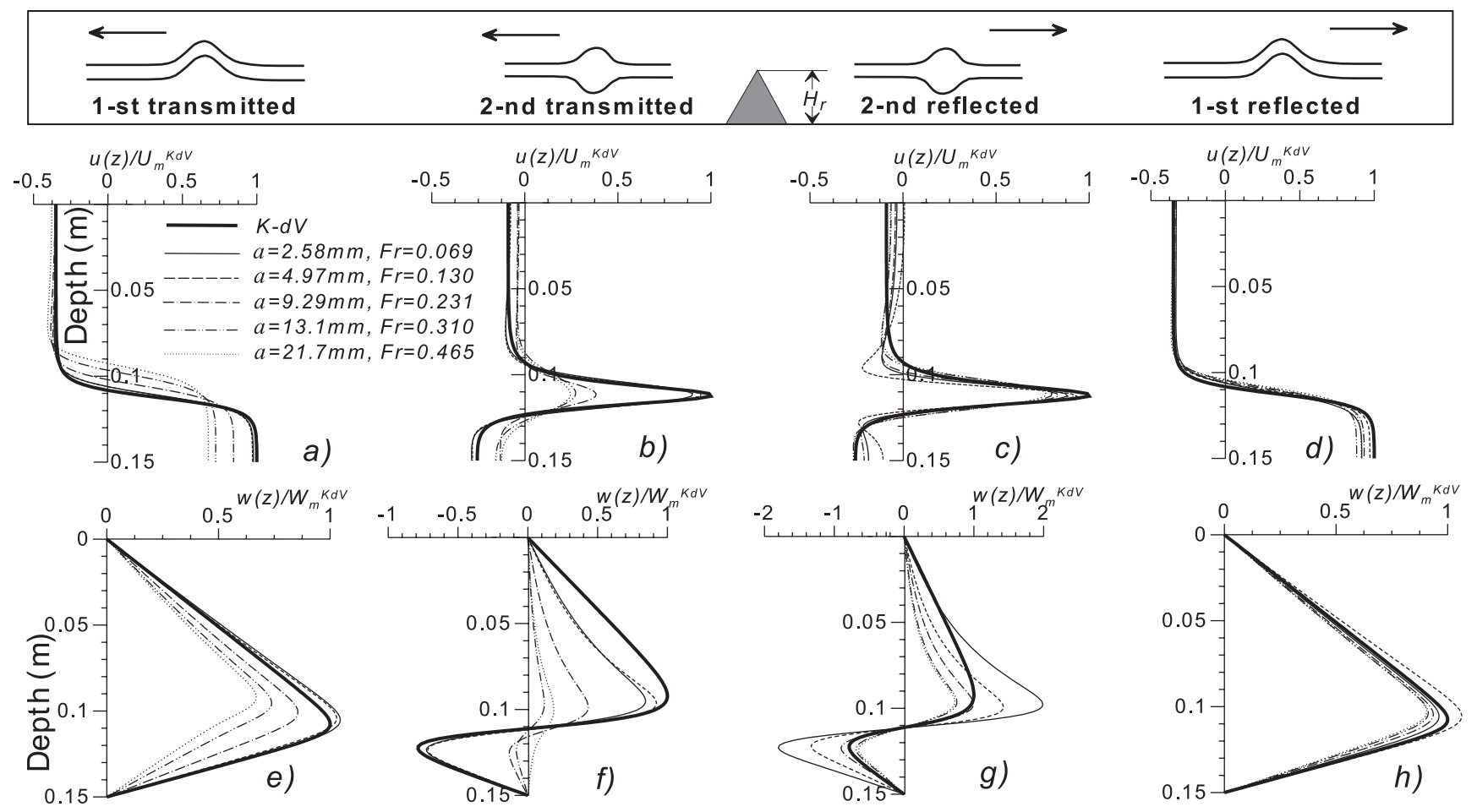

Fig. 14. Vertical profiles of horizontal (a)-(d) and vertical (e)-(h) velocities of transmitted and reflected waves plotted for several values of the amplitude $a$ of incident wave. Profiles were normalised with the maximum values of the appropriate K-dV solitons having the same amplitude. First reflected and first transmitted waves are compared with the first mode K-dV solitons, second reflected and second transmitted waves with the second mode K-dV solitons. Normalized profiles of K-dV solitary waves are presented by thick solid lines. All other notations are shown in the figure.
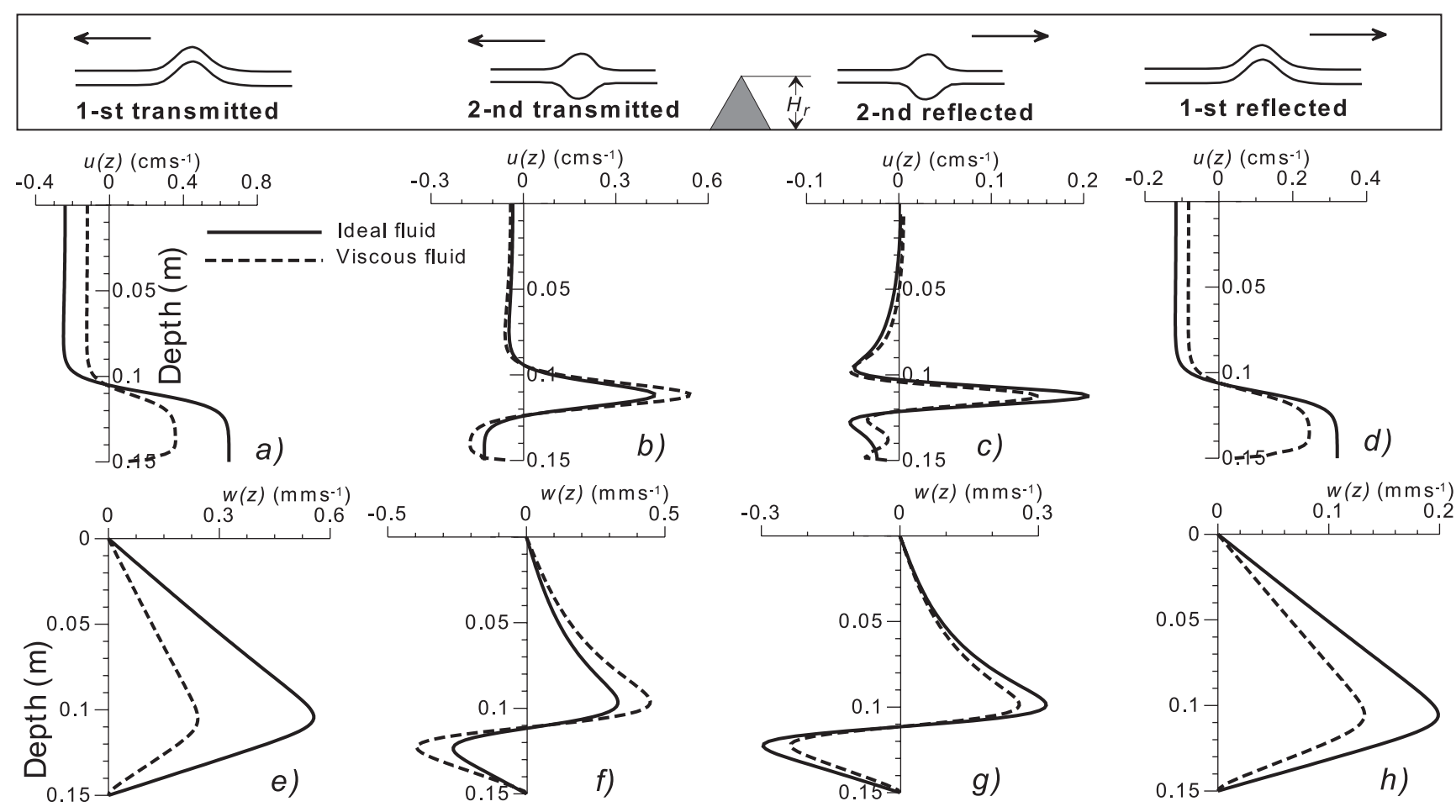

Fig. 15. Vertical profiles of horizontal (a)-(d) and vertical (e)-(h) velocities of transmitted and reflected waves calculated for $\mathrm{Fr}=0.13$ and $B=0.92$ in cases of ideal (solid lines) and viscous (dashed lines) fluids. All other notations are shown in the figure. 
reflected waves compared to the case with the slip boundary condition. Estimations from the laboratory experiments are a $35 \%$ decay of the wave amplitude at a distance of $8.5 \mathrm{~m}$. The remarkable difference in the rate of attenuation of the reflected and transmitted waves can be explained by the different amplitudes of these waves. The first transmitted wave is almost twice as large and substantially shorter than the first reflected wave (see Fig. 13).

A further interesting finding is a more remote influence of the dissipation on the characteristics of the second transmitted and reflected waves. Comparison of the vertical profiles for ideal and viscous fluids in Fig. 15 illustrates this fact. The less pronounced discrepancy between the vertical profiles for secondary waves in the ideal and viscous fluids (panels b, c, $f$ and $g$ in Fig. 15) compared to that for the first reflected and transmitted waves (panels a, e, $\mathrm{d}$ and $\mathrm{h}$ in Fig. 15) can be explained as follows: First, for a given elapsed time the second mode solitary waves, being three times slower, propagate a shorter distance from the source of their generation (from the sill) than first mode waves. They passed $2.5 \mathrm{~m}$ (transmitted) and $1.6 \mathrm{~m}$ (reflected) against $8.7 \mathrm{~m}$ and $8.4 \mathrm{~m}$ for the first mode waves. Second, the conditions of wave interaction with the sill in ideal and viscous fluids are somewhat different. When a bottom boundary layer is present the maximum of the horizontal velocity is displaced from the bottom to a position closer to the pycnocline. For this reason its vertical displacements over the sill are more pronounced; this fact leads to a more effective generation of second mode waves. For example, in the present case, the second transmitted wave has a $20 \%$ lager amplitude for the viscous fluid than the inviscid fluid.

These results on the solitary wave's damping rate are valid for the conditions of the laboratory experiments. Obviously they can not be in agreement with estimations valid for oceanic conditions, not even qualitatively. This is not surprising because real oceanic internal solitary waves have essentially different spatial and temporal scales and propagate in a medium with other coefficients of vertical and horizontal viscosity. For oceanic waves, such estimations of the damping rate obtained from the laboratory experiments constitute overestimations. This problem of attenuation of oceanic solitary waves was discussed, for instance, in a paper by Bogucki and Garret (1993) dealing with the question why such waves propagate such long distances (several hundreds of kilometres from the source of their generation). They do this only because dissipation is small.

\section{Summary and conclusions}

In this paper, experiments were described, that were performed in a $10 \mathrm{~m}$ long horizontal channel, on baroclinic wave propagation in a two-layered fluid system with an upper fresh-water and a lower salt-water layer. Because of the diffusive nature of the interface, this fluid system gives rise to the existence of higher order baroclinic modes not present in a two-layered system of constant density layers with a sharp interface between the two. The experimental setup was such that a single solitary wave, riding on the interface and corresponding to the baroclinic wave of the perfect twolayered system, was split into pairs of reflected and transmitted soliton-type waves representing the first mode wave of the layered system with diffusive interface (and corresponding to the wave of the perfect two layered system) plus a second mode due to the finite thickness of the interface. Because the interface thicknesses in the experiments were considered small, the observability of this second slow wave was not expected.

A numerical model, based on the two-dimensional NavierStokes equations in the Boussinesq approximation, was used to study the interaction of the first mode internal solitary wave with a sill. The basic goal of the numerical runs was to reproduce the laboratory experiments and to obtain an answer to the question which remained as a result of the laboratory experiment (and posed as a hypothesis) about the possibility of generation of second mode solitary waves during the interaction of the first mode internal solitons with a bottom obstruction. A unique answer to this question can be obtained from the comparison analysis of the excited solitary waves with the exact analytical solution of the K-dV equation. Such a solution was found for a three-parametric family of curves (7) which describes smooth pycnocline and approximates the laboratory law of vertical density distribution very well. Thus, the computations attempt to provide a "bridge" between the existing experimental results and the analytical predictions from the weakly nonlinear theory.

The structure and properties of the reflected and transmitted solitary waves are studied for a wide range of amplitudes of the incoming wave $(0<F r<0.5)$ and height of the underwater obstacle (blocking parameter $0<B<1.1$ ). Detailed analysis of the horizontal and vertical structures of the transmitted and reflected waves and comparison with the analytical stationary solution (9) of the K-dV equation, showed that in the wide range of controlling parameters $F r$ and $B$, the first reflected and transmitted waves of elevation, can be interpreted as a first mode internal solitary wave whose characteristics are close to the $\mathrm{K}-\mathrm{dV}$ solitons. Only small discrepancies arise for the first reflected waves in cases where their amplitudes are small. Such situations occur for sills with blocking parameter $B<0.6$ when the scattering of the incoming wave over the obstacle is relatively inefficient. Having large wavelengths (the same order as the wave tank length), weak reflected solitary waves must pass an additional distance of several tens of wavelengths (that was impossible under the conditions of our laboratory and numerical experiments) for a separation from another wave tail and for the formation of a K-dV soliton.

An additional discrepancy from K-dV solitons was found for first transmitted waves at $F r>0.2$. This disagreement increased with the growth of the amplitude of the incoming wave and was connected with the well known fact of the limited range of applicability of the weakly nonlinear theories. Large amplitude solitary internal waves must be considered in the frame of a fully nonlinear system of equations (1), (2). 
However, for solitary waves of relatively small and intermediate amplitudes $(F r<0.2)$ the coincidence was quite good.

Computed results have shown that the blocking parameter $B$ (the height of the sill) basically controls the energy loss of the incoming wave and its transfer to the reflected and transmitted secondary waves but it has a weak influence on the modal and spatial structures of these waves. At the same time the structures of the second reflected and transmitted wave (propagating approximately three times slower than and behind the first mode leading waves) were very sensitive to the amplitude of the incoming wave. At small Froude numbers $(F r<0.2)$, the second transmitted wave is treated as the second mode $\mathrm{K}-\mathrm{dV}$ soliton whereas the second reflected wave was in fact a dispersive packet of shorter waves.

This situation is valid at small Froude numbers $(\mathrm{Fr}<$ 0.2 ) but reverses with an increase of the amplitude. The second reflected waves at $\mathrm{Fr}>0.3$ fit the analytical K$\mathrm{dV}$ profile substantially better. At the same time the dependence of the characteristics of the second transmitted waves on the Froude number is the opposite. With an increase of the amplitude of the incoming wave its energy behind the sill is more effectively transferred into a dispersive first-modewave train accompanying the leading solitary wave than into second-mode-solitary waves (see Fig. 14). The second reflected wave in this case represents the extensive bulge of a pycnocline (wider than the K-dV soliton).

Computed results show that the wave characteristics are sensitive to the wall friction and internal friction. It was found that the wave amplitude decays exponentially with distance during the propagation of the internal wave. At the same time, the presence of a bottom boundary layer can lead to a more effective generation of second-mode-solitary waves due to the fact that, with the presence of a bottom boundary layer, the maximum of the horizontal velocity is displaced from the bottom to a position closer to the pycnocline. For this reason, its vertical displacements over the sill are more pronounced and this fact leads to a more effective generation of second mode waves.

The above experimental and theoretical results have been obtained for conditions of a small-scale laboratory experiment. However, they are also useful for the understanding of the dynamics of real oceanic solitary waves whose space and temporal scales are several orders larger. The generation of such waves is quite a regular feature at many sites, such as those excited by tidal flow over the shelf break, over ridges in fjords or wind events in long narrow lakes. Large amplitude internal solitary waves of permanent form can propagate large distances from the source of generation before encountering any further significant variations of the bottom topography (toward shore or in regions wherein the depth changes dramatically). We considered here one aspect of this problem, the scattering of a solitary wave by a simple triangular shape as an underwater obstacle; thus showed the possibility of generation of second mode internal solitary waves. Also, by interaction with nonhomogeneities of the medium, solitary waves can break down and generate instabilities, vertical and horizontal mixing, excitement of vortices and secondary waves. Such mechanisms may be very effective for mixing nutrient-rich waters from the bottom to the biologically active upper layers.

Acknowledgements. We thank Holger Hüttemann for the invaluable help and fruitful cooperation. This work was financially supported by the Deutsche Forschungsgemeinschaft.

\section{References}

Apel, J. R., Holbrock, J. R., Liu, A. K., and Tsai, J. J., The Sulu Sea internal soliton experiment, J. Phys. Oceanogr., 15, 1625-1651, 1985.

Apel, J. R., Ostrovsky, L. A., and Stepanyants, Yu. A., Internal solitons in the ocean, Tech. rep. MERCJRA0695, 70 pp, [Available from Milton S. Eisenhower Research Centre, Applied Physics Laboratory, The John Hopkins University, Johns Hopkins Rd., Laurel, MD 20707], 1995.

Bogucki, D. and Garret, C., A simple model for the shear-induced decay of an internal solitary wave, J. Phys. Oceanogr., 23, 17671776, 1993.

Diebels, S., Schuster, B., and Hutter, K., Nonlinear internal waves over variable topography, Geophys. Astrophys. Fluid Dynamics, 76, 165-192, 1994.

Farmer, D. M., Observations of long nonlinear waves in lake, J. Phys. Oceanogr., 8, 63-73, 1978.

Gerkema, T. and Zimmerman, J. T. F., Generation of nonlinear internal tides and solitary waves, J. Phys. Oceanogr., 25, 1081-1094, 1994.

Halpern, D., Observations of short period internal waves in Massachusetts Bay, J. Mar. Res., 29, 116-132, 1971.

Haury, J. M., Briscoe, M. G., and Orr, H. H., Tidally generated internal wave packets in the Massachusetts Bay, Nature, 278, 312317, 1979.

Helfrich, K. R., Internal solitary waves breaking and ru-up on a uniform slope, J. Fluid Mech., 243, 133-154, 1992.

Helfrich, K. R. and Melville, W. K., On long nonlinear waves over slope-shelf topography, J. Fluid Mech., 167, 285-308, 1986.

Helfrich, K. R., Melville, W. K., and Miles, J. W., On interfacial solitary waves over slowly varying topography, J. Fluid Mech., 149, 305-317, 1984.

Holloway, P. E., Internal hydraulic jumps and solitons at the shelf break region on the Australian West Shelf, J. Geoph. Res., 92, 5405-5416, 1987.

Holloway, P. E., Pelinovsky, E., Talipova, T., and Barnes, B., A nonlinear model of internal tide transformation on the Australian North West shelf, J. Phys. Oceanogr., 27, 871-896, 1997.

Hüttemann H. and Hutter K., Baroclinic solitary water waves in a two-layered fluid system with diffusive interface, Experiments in Fluids, 30, 317-326, 2001.

Kaup, D. J. and Newell, A. C., Solitons as particles, oscillators, and in slowly changing media: a singular perturbation theory, Proc. R. Soc. Lond., A361, 413-446, 1978.

Krauss, W., Interne Wellen, Gebrüder Bornträger, BerlinNikolassee, 1966.

Lamb, K. G., Numerical experiments of internal wave generation by strong tidal flow across a finite amplitude bank edge, J. Geophys. Res., 99, 843-864, 1994.

Lamb, K. G. and Yan, L., The evolution of internal wave undular bores: comparison of fully nonlinear numerical model with 
weakly nonlinear theory, J. Phys. Oceanogr., 26, 2712-2734, 1996.

Liu, A. K., Analysis of nonlinear internal waves in the New-York Bight, J. Geophys. Res., 93, 12317-12329, 1988.

Liu A. K., Chang, Y. S., Hsu, M.-K., and Lang, N. K., Evolution of nonlinear internal waves in the East and South China Seas, J. Geophys. Res., 103, 7995-8008, 1998.

Long R. R., Some aspects of the flow of stratified fluids, Tellus, 5, 42-58, 1953.

Marchuk, G. I., Numerical methods in weather prediction, Academic Press, 1974.

Maurer, J., Hutter, K., and Diebels, S., Viscous effects in internal waves of a two-layered fluid with variable depth, Eur. J. Mech., B/Fluids, 15, N4, 445-470, 1996.

Maxworthy, T., A note on the internal solitary waves produced by tidal flow over three-dimensional ridge, J. Geophys. Res., 84, 338-346, 1979

Michallet, H. and Barthelemy, E., Experimental study of solitary waves, J. Fluid Mech., 366, 159-177, 1998.

Michallet, H. and Ivey, G. M., Experiments on mixing due to internal solitary waves breaking on uniform slopes. J. Geophys. Res., 104, 13467-13477, 1999.

Miyata, M., An internal solitary waves of large amplitude, La Mer, 23, 43-48, 1985.

Miyata, M., Long internal waves of large amplitude, in: K. Horikawa, H. Maruo (Eds.), Nonlinear water waves, Springer Verlag, New-York, 23, 399-406, 1988.

Mortimer C. H., Water movements in lakes during summer stratification; evidence from the distribution of temperature in Windermere, Phil. Trans. R. Soc. London, 236B, 355-404, 1952.

Osborne, A. R. and Burch, T. I., Internal solitons in the Andaman Sea, Nature, 208, 451-469, 1980.
Ostrovsky, L. A. and Stepanyants, Yu. A., Do internal solitons exist in the ocean?, Rev. Geophys., 27, 293-310, 1989.

Sabinin, K. D., Internal wave packets over the Maskaren ridge, Izv. Acad. Sci. USSR, Atmos. Oceanic Phys., 28, 625-633, 1992.

Salusti, E., Laskrator, A., and Nittis, K., Changes of polarity in marine internal waves: Field evidence in Mediterranean Sea Ocean Modell., 82, 53-82, Hook Inst., Univ. of Oxford, Oxford, England, 1989.

Salvade G., Zambone, F., and Barbieri, A., Three-layer model of the north basin of the Lake of Lugano, Ann. Geophysicae, 5, 247 254, 1987.

Sandstrom, H. and Elliott, J. A., Internal tide and solitons on the Scotian Shelf: A nutrient pump at work, J. Geoph. Res., 89, 6415-6426, 1984.

Stevens, C., Lawrence, G., Hamblin, P., and Carmack, E., Wind forcing of internal waves in a long narrow stratified lake, Dyn. Atmos. Oceans, 24, 41-50, 1996.

Terez, D. E. and Knio, O. M., Numerical simulations of largeamplitude internal solitary waves, J. Fluid Mech., 362, 53-82, 1998.

Thorpe, S. A., Asymmetry of the internal wave seiche in the Loch Ness, Nature, 231, 306, 1971.

Vlasenko, V. I., Multi-modal soliton of internal waves, Atmos. Oceanic Phys., 30, 161-169, 1994.

Vlasenko, V. I., Brandt, P., and Rubino, A., On the structure of large-amplitude internal solitary waves, J. Phys. Oceanogr., 30, $142-155,2000$

Wessels, F. and Hutter, K., Interaction of internal waves with a topographic sill in a two-layered fluid, J. Phys. Oceanogr., 26, 5-20, 1996.

Wunsch C. and W. H. Munk, W. H., Abyssal recipe II, Deep-Sea Res., 45, 1976-2009, 1998. 